\title{
Diffuse polarized emission associated with the Perseus cluster
}

\author{
A. G. de Bruyn ${ }^{1,2}$ and M. A. Brentjens ${ }^{2,1}$
}

\author{
1 ASTRON, PO Box 2, 7990 AA Dwingeloo, The Netherlands \\ e-mail: ger@astron.nl \\ 2 Kapteyn Astronomical Institute, University of Groningen, PO Box 800, 9700 AV, Groningen, The Netherlands
}

Received 4 March 2005 / Accepted 8 July 2005

\section{ABSTRACT}

We report on full-polarization radio observations of the Perseus cluster (Abell 426) using the Westerbork Synthesis Radio Telescope (WSRT) at wavelengths from 81-95 cm. We detect faint, very extended polarized emission throughout the cluster region. We have employed a novel technique, Rotation Measure synthesis (Brentjens \& de Bruyn, 2005, A\&A, 441, 1217) to unravel the polarization properties of the emission across the full field of view. We detect polarized emission over a wide range of RM from about 0 to $90 \mathrm{rad} \mathrm{m}^{-2}$. Low $\mathrm{RM}$ emission $(\mathrm{RM}<$ $15 \mathrm{rad} \mathrm{m}^{-2}$ ) is attributed to the local Galactic foreground. It has a chaotic structure with smooth changes in polarization angle on scales of the order of $10^{\prime}-30^{\prime}$, not unlike those seen by Haverkorn et al. (2003a, A\&A, 403, 1045) at the same frequencies. Emission at values of RM > $30 \mathrm{rad} \mathrm{m}^{-2}$ on the other hand, shows organized structures on scales up to a degree and displays rapidly fluctuating polarization angles on scales of the synthesized beam. A Galactic foreground interpretation for the high RM emission can not be ruled out, but appears extremely implausible. WSRT observations at $21 \mathrm{~cm}$ of the RM of a dozen discrete sources surrounding the Perseus cluster indicate a smooth large-scale gradient in the Galactic foreground RM. The diffuse structures have a clear excess RM of about $40 \mathrm{rad} \mathrm{m}^{-2}$ relative to these distant radio galaxies. This excess Faraday depth, the generally good spatial association with the cluster and the different morphology of the high RM emission, compared to the genuine Galactic foreground emission, all point to an association of the high RM emission with the Perseus cluster. The polarized emission reaches typical surface brightness levels of $0.5-1 \mathrm{mJy}$ per $2^{\prime} \times 3^{\prime}$ beam and must be rather highly polarized ( $\left.\approx 20 \%\right)$. Due to dynamic range limitations and lack of sensitivity to large-scale structure we have not yet detected the corresponding total intensity. Most of the polarized emission, located at distances of about $1^{\circ}$ from the cluster centre, appears too bright, by about $1-2$ orders of magnitude, to be explainable as Thomson scattered emission of the central radio source off the thermal electrons in the cluster. However, this remains a viable explanation for the highly polarized $21 \mathrm{~cm}$ emission from the inner $10^{\prime}-20^{\prime}$ and part of the $81-95 \mathrm{~cm}$ emission. The bulk of the emission associated with the Perseus cluster may instead be related to buoyant bubbles of relativistic plasma, probably relics from still active or now dormant AGN within the cluster. A lenticular shaped structure, referred to as the lens, and measuring $0.5-1 \mathrm{Mpc}$ is strikingly similar to the structures predicted b Enßlin et al. (1998, A\&A, 332, 395). At the western edge of the cluster, we detect very long, linear structures that may be related to shocks caused by infall of gas into the Perseus cluster along the Perseus-Pisces filamentary structure of the cosmic web.

Key words. galaxies: clusters: general - galaxies: active - polarization - magnetic fields - techniques: image processing radio continuum: general

\section{Introduction}

Clusters of galaxies play a crucial role in many fields of extragalactic research. Located at the crossroads of the filamentary structures known as the cosmic web, they point to the areas with the deepest potential wells as predicted in the standard CDM model for structure formation in the Universe. In addition to the dominant dark matter and the large numbers of visible galaxies, clusters also contain enormous reservoirs of diffuse hot gas. This gas is visible directly through their X-ray emission, and indirectly through their dynamical effects on galaxies, such as stripping, and the shaping of head-tail radio sources.

Because of their deep potential wells, clusters continue to accrete gas from their surroundings. At the cluster-IGM interface this should lead to (mild) shocks (Burns 1998) which might be observed through enhanced X-ray and radio emission.
In the cluster Abell 3667 infall indeed appears to be responsible for the generation of very large radio and polarized source structures (Rottgering et al. 1997; Johnston-Hollitt 2004).

The large-scale distribution of the hot gas can in principle be derived from the X-ray surface brightness profiles. However, there are two caveats. First, due to the quadratic dependence of X-ray emission on gas density, this distribution depends on the level of clumpiness in the medium. Furthermore, temperatures below a few times $10^{6} \mathrm{~K}$ also lead to reduced X-ray emission and therefore might lead to an underestimate of the amount of gas. Recent XMM observations (Kaastra et al. 2003) indeed suggest that significant amounts of lower temperature gas exist at the outskirts of clusters. These observations may have a direct bearing on the question "Where are the missing baryons in the universe?" (Cen \& Ostriker 1999). An independent linear probe of the gas density would be helpful to 
unambiguously deduce the gas distribution. The scattering of the $2.7 \mathrm{~K}$ microwave background photons by the cluster electrons, the Syunyaev-Zeldovich effect, is such a method. Another linear probe of the gas density is provided through Thomson scattering of radio emission located within the cluster (Syunyaev 1982; Wise \& Sarazin 1990). This method, which has a fair number of assumptions built in, has a better chance of detection in low redshifts clusters because of confusion problems. Until now no unambiguous identification of Thomson scattering in clusters has been made. Very high dynamic range $21 \mathrm{~cm}$ continuum observations with the WSRT (de Bruyn 1995) may have detected such emission and were the original motivation for the observations described in this paper.

Recent radio studies of clusters have drawn attention to another interesting aspect of clusters, namely the role played by radio loud $\mathrm{AGN}$ in the thermal balance of the intracluster medium. Combined radio and X-ray images of the inner parts of the Perseus and other rich clusters with strong radio sources (Fabian et al. 2003; Clarke et al. 2004) clearly show how the high pressure radio lobes of the central radio sources are the cause of cavities in the X-ray surface brightness.

If AGN activity in the radio band is episodic (Schoenmakers et al. 2000), clusters should contain many relic, or fossil, radio sources. Such sources have now been detected in several clusters (Enßlin et al. 1998; Govoni et al. 2001, 2005). Enßlin \& Gopal-Krishna (2001) show that orphan radio relics would slowly rise in the cluster atmosphere as a result of buoyancy forces. The adiabatic energy losses (on both particles and magnetic field) would then render these relics effectively invisible at $\mathrm{GHz}$ frequencies. These "bubbles" could then "hibernate" for periods of several Gyrs and be invisible except at extremely low frequencies. These relics could be "woken up" by the shocks associated with the inflow of gas from the surroundings.

In this paper we describe observations of polarized radio emission from the Perseus cluster which provide a new tool to study the three components of the diffuse intracluster medium: thermal gas, relativistic gas, and magnetic fields. Low frequency radio polarization data may convey information about extremely low density regions if they are pervaded by a magnetic field. Such measurements, however, are complicated by instrumental and astrophysical effects which have limited their application in the past. First, off-axis instrumental polarization of radio telescopes is generally quite severe at low frequencies. Using mosaicking techniques to image large fields will alleviate this to a large degree. Second, ionospheric Faraday rotation can rotate polarization angles by up to hundreds of degrees during the synthesis time at a frequency around $325 \mathrm{MHz}$. This can be limited by observing at night and using GPS-based and ionosonde-based estimates of the ionospheric electron content. Third, the percentage polarization of radio sources is generally low at low frequencies as a result of internal depolarization due to Faraday rotation within the emission regions (Strom \& Conway 1985; Conway \& Strom 1985) and as a result of beam depolarization. When observing sources with a large rotation measure, or Faraday depth (Burn 1966), a fourth complication arises: bandwidth depolarization.
In order for standard polarization analysis (e.g. RM-fitting) to be meaningful a minimum $\mathrm{S} / \mathrm{N}$ of about 3 is required in the linearly polarized signals for each frequency. Increasing the bandwidth to improve the $\mathrm{S} / \mathrm{N}$, however, conflicts with the requirement to limit the bandwidth to avoid bandwidth depolarization. For example, observing a polarized source at $350 \mathrm{MHz}$ with a bandwidth of $80 \mathrm{MHz}$, limits the allowed RM to about $5 \mathrm{rad} \mathrm{m}^{-2}$ before Faraday rotation quenches all polarized signals by an order of magnitude. To obtain good sensitivity yet avoid bandwidth depolarization requires a wideband correlator with a large number of channels. As of April 2002 the Westerbork Synthesis Radio Telescope (WSRT) can cross correlate, in full polarization, data from all 14 telescopes in up to 1024 spectral channels over an $80 \mathrm{MHz}$ band. Employing a technique, which we have called Rotation Measure synthesis (Brentjens \& de Bruyn 2005) and which is briefly described below, then provides an elegant and powerful method to allow noise limited polarimetry using a wide frequency band with arbitrarily low $\mathrm{S} / \mathrm{N}$ ratio in each individual narrow frequency channel.

A powerful feature of RM-synthesis is the ability to search for weakly polarized emission over a wide field of view. A simple version of the method was developed by de Bruyn (1996) for the old WSRT 8 channel continuum backend. It was implemented in the WSRT reduction package NEWSTAR and first applied to data from the highly polarized millisecond pulsar PSR J0218+4232 (Navarro et al. 1995). The method will be described briefly in Sect. 4 but a full derivation, discussion and analysis of its properties is presented in a companion paper (Brentjens \& de Bruyn 2005).

The outline of this paper is as follows. In Sect. 2 we present the deep WSRT observations of the Perseus cluster motivating this paper. Section 3 describes the total intensity and polarization calibration. Section 4 describes how the basic data products were made. Section 5 discusses instrumental artefacts and how they can be distinguished from the interesting astronomical signals. Sections 6 and 7 discuss the nature and location of the various types of polarized structures. Section 8 presents our interpretation of the features that we attribute to the Perseus cluster. Some puzzles and planned future observations are presented in Sect. 9 while Sect. 10 concludes.

\section{Observations}

The observations were conducted with the WSRT. The array consists of fourteen $25 \mathrm{~m}$ dishes on an east-west baseline and uses earth rotation to fully synthesize the uv-plane. For full imaging over the whole primary beam it takes six array configurations in which the four movable telescopes are stepped at $12 \mathrm{~m}$ increments (i.e. half the dish diameter) with the shortest spacing running from $36 \mathrm{~m}$ to $96 \mathrm{~m}$. This provides continuous uv-coverage with interferometer baselines ranging from 36 to $2760 \mathrm{~m}$. The $12 \mathrm{~m}$ increment creates an elliptic grating lobe with a radius in Right Ascension of about $4^{\circ}$ from the phase centre, which places it well beyond the $10 \mathrm{~dB}$ point of the primary beam which measures 2.4 full width at half power. Self confusion is therefore not a problem within the $-5 \mathrm{~dB}$ point of 
Table 1. Some parameters of the $92 \mathrm{~cm}$ WSRT observations. Dates are formatted as yyyy/mm/dd hh:mm:ss.

\begin{tabular}{lrll}
\hline \hline Observation ID & 9-A (m) & Start date (UTC) & End date (UTC) \\
\hline 10208707 & 72 & $2002 / 12 / 0216: 06: 20$ & $2002 / 12 / 0304: 04: 50$ \\
10208767 & 36 & $2002 / 12 / 0615: 50: 30$ & $2002 / 12 / 0703: 49: 00$ \\
10208819 & 96 & $2002 / 12 / 1015: 34: 50$ & $2002 / 12 / 1103: 33: 20$ \\
10208868 & 84 & $2002 / 12 / 1215: 27: 00$ & $2002 / 12 / 1303: 25: 30$ \\
10208937 & 48 & $2002 / 12 / 1415: 19: 10$ & $2002 / 12 / 1503: 17: 40$ \\
10208997 & 60 & $2002 / 12 / 1715: 07: 20$ & $2002 / 12 / 1803: 05: 50$ \\
\hline
\end{tabular}

the primary beam. The angular resolution in Right Ascension is about $0.8^{\prime}$. The half power beam width, grating lobe and resolution all correspond to a frequency of $350 \mathrm{MHz}$, in the middle of the observing band, and scale inversely with frequency. The grating lobe and the angular resolution furthermore scale with $1 / \sin (\delta)$ in the declination direction. Basic observational data are collected in Table 1. The pointing and phase centre of the telescope was directed towards (B1950.0): $\mathrm{RA}=03^{\mathrm{h}} 15^{\mathrm{m}}$, Dec $=+41^{\circ} 15^{\prime}$, which is located between the three dominant radio sources in the Perseus cluster. This position was chosen to be identical to that of previous WSRT observations described in Sijbring (1993) to allow a comparison between the datasets.

The feed/receiver is part of the new Multi Frequency FrontEnd package (http://www.astron.nl/wsrt/) and covers the frequency range from about $310-390 \mathrm{MHz}$. These frequencies correspond to wavelengths from 78-97 $\mathrm{cm}$ but for historical reasons, and brevity, we will continue to refer to this as the $92 \mathrm{~cm}$ band. The $92 \mathrm{~cm}$ band is usually largely free of radio frequency interference (RFI) during evening, nighttime and weekend observing at the location of the WSRT. The full band can be completely covered by the new wide band correlator which can process 8 independently tunable bands of $10 \mathrm{MHz}$. Each band is covered by 64 channels in 4 cross-correlations to recover all Stokes parameters. We used Hamming tapering in the lag-to-frequency transform yielding an effective spectral resolution of $0.31 \mathrm{MHz}$. Hamming taper, rather than Hanning, was used to lower the distant spectral side lobe level (Harris 1978). The channel separation was $0.156 \mathrm{MHz}$. The frequencies of the 8 bands were centred at 319, 328, 337, 346, 355, 365,374 , and $383 \mathrm{MHz}$. A gap at $360 \mathrm{MHz}$ was introduced to avoid local RFI. Gibbs ringing at the video edge of the band and the increased (digital) noise at the upper filtered end of the band led to useful data in 56 (out of 64) channels of each $10 \mathrm{MHz}$ band (channels 3-58). Unfortunately, serious external RFI was encountered during several hours into the evening at the start of the observations. In several $10 \mathrm{MHz}$ bands these intense but narrow RFI signals "ring" through the whole band, despite our use of a Hamming taper. By combining pairs of odd-even channels in the subsequent processing this ringing could be eliminated and the final analysis was therefore done using 28 channel pairs for each $10 \mathrm{MHz}$ band. Henceforth we will refer to such a channel pair as a channel. It has an effective spectral resolution of about $0.4 \mathrm{MHz}$. The RFI frequently spoiled the total power monitoring data preventing a precise absolute calibration of the data. This absolute calibration was therefore done using the 1984 WSRT observations at $327 \mathrm{MHz}$ (Sijbring 1993).
Full syntheses with the WSRT usually need not be interrupted during a 12 hour track. Because of its equatorial mount - no change in parallactic angle - it also suffices to observe only one polarized and one unpolarized calibrator to calibrate instrumental leakages. Every observation was bracketed by two pairs of calibrators, one polarized and one unpolarized: 3C 345 and 3C 48 were observed before the target, while 3C 147 and the bright, highly polarized eastern hot spot in DA 240 were observed at the end. The calibrator observations lasted $30 \mathrm{~min}$. The time resolution in the data was $30 \mathrm{~s}$, which was generally sufficient to fully sample ionospheric phase fluctuations without serious phase decorrelation effects. This time sampling was also sufficient to avoid time smearing for sources at the outer edge of the field.

The system temperature of the WSRT at $350 \mathrm{MHz}$ towards the Perseus cluster is about $125-150 \mathrm{~K}$. With a net integration time of $72 \mathrm{~h}$ this should result in a thermal noise of about $20 \mu \mathrm{Jy}$ for an effective bandwidth of $8 \times 8.7 \mathrm{MHz}$. However, we did not reach this level for the following reasons. Calibration problems did not allow us to include the upper two frequency bands (374 and $383 \mathrm{MHz}$ ). Due to serious malfunctioning in two telescopes/receivers (RT0 and RTC), one of which (RTC) provides half of the long baselines in the array, we decided to use only the inner half of the array in the final analysis. This also helped to render the very faint but very extended polarized emission visible. To avoid grating lobes, and the inherent self confusion once emission fills the whole primary beam, we did not use natural weighting in the imaging (i.e. the redundant baselines were not included in the imaging process). Hence the final image cubes were made with only 18 of the maximum 91 baselines for each $12 \mathrm{~h}$ synthesis. This resulted in a synthesized beam of $2.0^{\prime} \times 3.0^{\prime}$. Henceforth, when quoting flux densities, we will always refer to this beam size unless otherwise specified.

The theoretically achievable noise level should increase by a factor 2.5 to $50 \mu \mathrm{Jy}$ (this corresponds to $50 \mathrm{mK}$ brightness temperature). The final noise level, after flagging about $25 \%$ of the data that were affected by faint RFI or backend problems, was about $70 \mu \mathrm{Jy}$ per beam. This, however, is still much lower than the classical confusion noise of about $1.5 \mathrm{mJy}$ for this frequency and angular resolution and presents by far the deepest low frequency image ever made.

\section{Data reduction}

\subsection{Total intensity calibration}

The Perseus cluster was first observed with the WSRT at $327 \mathrm{MHz}$ in 1984 (Sijbring 1993). The cluster contains a total 


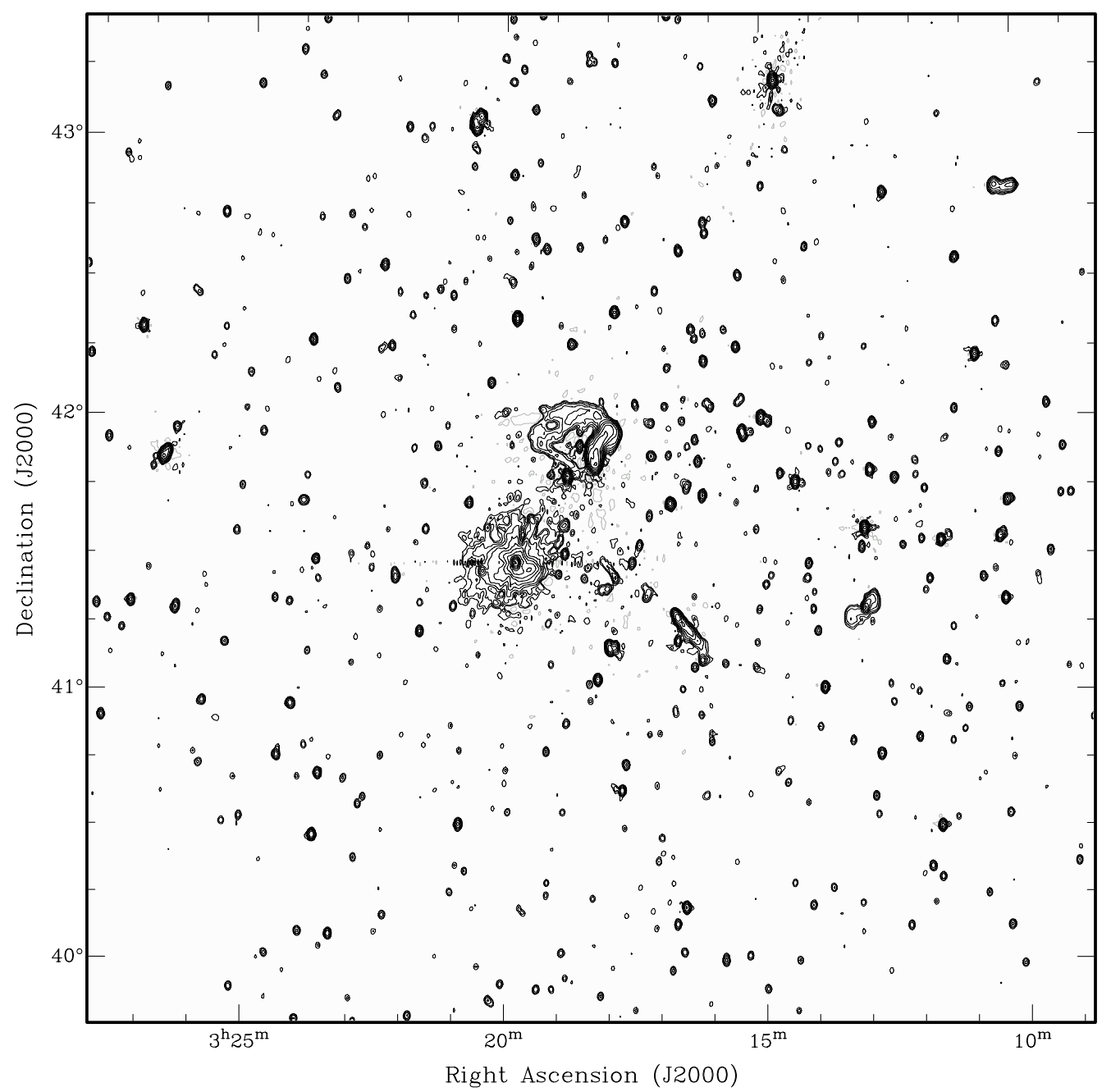

Fig. 1. Total intensity image of the Perseus cluster, observed with the WSRT in 2002, using data from band 3. Contour levels are at $-2,2,4,8$, $16, \ldots, 16384$ mJy beam $^{-1}$.

of about $50 \mathrm{Jy}$ of diffuse emission, concentrated in NGC 1275 (3C 84) and NGC 1265 (3C 83.1), and an unusually rich concentration of bright $4 \mathrm{C}$ sources at the edge of the primary beam (4C 41.08, 4C 42.09, 4C 43.09). The complexity of the brightness distribution necessitated several non-standard steps in the processing which was done using the WSRT-tailored NEWSTAR reduction package. Initially the total intensity selfcalibration of the data did not converge satisfactorily. This we attributed to the absence of a sufficiently accurate initial amplitude calibration (no system temperature corrections were applied) in the starting model. This is a well-known problem in WSRT observations of very complex fields, and is fundamentally due to the 1-dimensional nature of the WSRT array. By using the redundancy constraints in the array (Wieringa 1992) we were able to generate an improved starting image which converged much better after self-calibration. Obviously bad data were edited at the start; more sophisticated flagging was done in an iterative fashion on the basis of the selfcal residuals. The total intensity image of the Perseus cluster is shown in Fig. 1. This image was made from 11 channels in band 3 (average frequency $348 \mathrm{MHz}$ ) and is shown at full resolution $\left(0.9^{\prime} \times 1.3^{\prime}\right.$ beam $)$. The noise level varies across the image from about $0.3-1.0 \mathrm{mJy}^{-1}$ beam ${ }^{-1}$ with the higher values in the inner $2^{\circ}-3^{\circ}$ of the imaged area surrounding $3 \mathrm{C} 84$ and $3 \mathrm{C} 83.1 \mathrm{~B}$. Compared to the peak intensity of $18 \mathrm{Jy}$ on $3 \mathrm{C} 84$ this represents a dynamic range of about 20000:1. The observed noise is only a factor 1.5-2 above the classical confusion noise level.

High dynamic range imaging at low frequency usually is limited by the phase stability of the ionosphere. In general the ionospheric phase fluctuations were rather modest during the 6 nights of the observation, with peak excursions on a $1.4 \mathrm{~km}$ baseline of about $30^{\circ}$ on timescales of 3-5 min. In several nights, however, we experienced periods of a few hours of much faster, temporally unresolved, scintillation related phase excursions. These phase variations point to small scale structure in the ionosphere and led to imaging problems, because they invalidate the standard self-calibration assumption of position invariant errors. These manifest themselves in the images as spiky patterns surrounding bright sources at the edge of the observed field (Note that the WSRT instantaneous response has a fan beam response which rotates clockwise from position angle $90^{\circ}$ to $270^{\circ}$ during the $12 \mathrm{~h}$ synthesis time). The most visible ionospheric problems are those surrounding 4C 43.09, which is located about 1:8 NNE from the phase 
centre. They are also visible around 4C 41.08, located about 1.6 east of the cluster centre. We also detect weak amplitude errors around these sources which are due to pointing problems. The results described in this paper are concerned only with the diffuse polarization in the inner $3^{\circ}$ of the cluster and are not affected by pointing. We have recently started to experiment with a new iterative "source-by-source" self-calibration procedure, called peeling in the context of LOFAR $^{1}$, to remove very bright sources with their own ionospheric phase and telescope complex gain solutions. Because this paper is mainly concerned with the polarized emission we will not elaborate on this procedure here any further.

\subsection{Polarization calibration}

The WSRT telescopes are equipped with a pair of orthogonal linear feeds. All four cross correlations between the incident signal are formed. The polarization calibration followed the procedures described in the papers by Hamaker et al. (1996) and Sault et al. (1996). The overall (on-axis) instrumental leakages, typically $1-2 \%$, were calibrated using the unpolarized calibrator $3 \mathrm{C} 147$. The off-axis polarization behaviour is much more complicated and is described in more detail in Sect. 5.1.

The instrumental polarization corrections were transferred to the polarized calibrator source DA 240 which is then used to phase align the two orthogonal linear polarizations. The eastern hot spot of DA 240, has about $600 \mathrm{mJy}$ of linearly polarized signal and its RM is about $+4 \mathrm{rad} \mathrm{m}^{-2}$. The final polarization corrections were then transferred to the target source. The "peeling" method alluded to above was also used to correct for the position and frequency dependent instrumental polarization. This was done on a channel-by-channel basis. Doing this for $6 \times 28=168$ channels made this a very time consuming process. For each channel we performed a full self-calibration as well as polarization calibration using the same starting model adjusted to take care of the different primary beam attenuations at the different frequencies. The self-calibration model included (instrumental) polarization for the brightest discrete sources. The "peeling" was done for 4 different sources (3C 84, $3 \mathrm{C} 83.1 \mathrm{~A}+3 \mathrm{C} 83.1 \mathrm{~B}, 4 \mathrm{C} 43.09$ and $4 \mathrm{C} 41.08)$ in succession. The full details of this procedure, which are not very relevant for the purpose of this paper which deals mainly with the polarization results, will be described elsewhere.

At the observed frequencies ionospheric Faraday rotation can sometimes be a problem, especially during day-time observing. We do not have a strongly polarized source within the Perseus field on which we can "self calibrate" the ionospheric Faraday rotation. We therefore made use of data from two near-simultaneous $12 \mathrm{~h}$ syntheses of NGC 891, observed for a different project during the 2-week observing campaign on the Perseus cluster. This field contains the highly polarized pulsar PSR J0218+4232 (Navarro et al. 1995). The pulsar's polarization angle revealed a Faraday rotation of about $60^{\circ}$ at the start decreasing to about zero after $4 \mathrm{~h}$ and remaining small for the remaining $8 \mathrm{~h}$ of the observation. The Faraday rotation occurred in the few hours before and just after sunset. Because

\footnotetext{
1 A name suggested by Jan Noordam.
}

the declination and epoch of the NGC 891 and Perseus observations were very similar we adjusted for the $1 \mathrm{~h}$ RA-difference and de-applied a polarization angle rotation of $45^{\circ}$ decreasing to zero during the first $3 \mathrm{~h}$ of all six $12 \mathrm{~h}$ Perseus cluster syntheses.

\section{From $Q-U$ image cubes to RM-cubes}

For the final polarization analysis we eliminated 42 channels from the available 168 because they showed faint traces of RFI or dynamic range problems related to specific telescopes. The remaining 126 images were carried through for further processing in RM-synthesis. The average noise level in the Stokes $Q$ and $U$ polarization images of a single channel was rather uniform and varied from about $0.7-0.8 \mathrm{mJy}$.

The wide range of frequencies in the data set implies that the primary beam width changes significantly across the band. At the mid-band frequency of $345 \mathrm{MHz}$ the half power beam width $(H P B W)$ measures about 1.22 . The WSRT primary beam is well approximated by a $\cos (c v r)^{6}$ function where $c$ is a constant equal to about 0.064, $v$ is the frequency in $\mathrm{MHz}$ and $r$ the radius from the pointing centre in degrees. For the polarized structures discussed in this paper, which are detected out to a radius of about 1.5 , the maximum reduction is about a factor 3.5 at the highest frequency of $370 \mathrm{MHz}$ used in the analysis. The differential attenuation between 315 and $370 \mathrm{MHz}$ at this distance from the pointing centre is still only a modest factor 1.4. We have not tried to correct the data for these differential effects. In a future paper we will return to the spectral properties of the polarized emission and, if we manage to recover them, the associated total intensity structures.

\subsection{Rotation-measure synthesis}

The basic idea behind rotation measure synthesis is that one derotates, for every pixel in each channel image, the $Q-U$ vectors in order to compensate for a certain assumed rotation measure. After derotation, the channel images are averaged. This procedure maximizes sensitivity to radiation at the assumed Faraday depth, because that emission is coherently added. All other emission will add only partly coherently, hence the sensitivity to emission not at the assumed Faraday depth is reduced. The essence of this procedure was already mentioned by Burn (1966). The procedure is not unknown in the pulsar community (Mitra et al. 2003; Weisberg et al. 2004). However, it is usually only applied to a single source or line-of-sight (Bower et al. 1999; Killeen 1998). de Bruyn (1996) applied the method to every pixel in WSRT $350 \mathrm{MHz}$ radio synthesis data of the field surrounding the galaxy NGC 891 and the highly polarized pulsar PSR J0218+4232, but with only 8 frequencies available the RM side lobes were rather high. Following the completion of the new broadband $(8 \times 10$ or $20 \mathrm{MHz}) 250000$ channel WSRT backend the method was expected to become much more powerful. A full description of RM-synthesis is presented in a companion paper (Brentjens \& de Bruyn 2005). Here we summarize the essential features of the method as applied to the Perseus cluster data. 
The derotation of the multi channel complex polarization images can be performed very efficiently in terms of computer time. One first computes the complex polarization $P=Q+\mathrm{i} U$ and multiplies with a complex phase factor to perform the rotation. Brentjens \& de Bruyn (2005) derive that a general inversion of the polarization as a function of wavelength squared is given by:

$\{F * R\}(\phi)=\frac{\int_{-\infty}^{+\infty} W\left(\lambda^{2}\right) P\left(\lambda^{2}\right) \mathrm{e}^{-2 i \phi\left(\lambda^{2}-\lambda_{0}^{2}\right)} \mathrm{d} \lambda^{2}}{\int_{-\infty}^{+\infty} W\left(\lambda^{2}\right) \mathrm{d} \lambda}$,

where $\phi$ is the Faraday depth,

$\phi=0.81 \int_{\text {there }}^{\text {here }} n_{\mathrm{e}} \boldsymbol{B} \cdot \mathrm{d} \boldsymbol{l}$,

and $F(\phi)$ is the emission as a function of Faraday depth, * denotes convolution, $R(\phi)$ the rotation-measure transfer function (RMTF), $\lambda_{0}^{2}$ is the square of the wavelength to which all vectors are derotated, and $W\left(\lambda^{2}\right)$ the sensitivity as a function of wavelength squared. This function is also known as the sampling function, or weight function. $F(\phi)$ is measured in $\mathrm{Jy}_{\text {beam }}{ }^{-1} \mathrm{rmtf}^{-1}$. For extended sources that are discrete in $\phi$, this corresponds to the polarized surface brightness. For sources that are extended with respect to $\phi$, it is the polarized surface brightness per RMTF beam width.

If the bandwidth of an individual channel is much less than the total bandwidth of the observation, we may approximate the weight function by a sum of $\delta$ functions. That enables us to discretize Eq. (1):

$$
\{F * R\}\left(\phi_{k}\right)=\frac{\sum_{i=1}^{N} w_{i} P_{i} \mathrm{e}^{-2 \mathrm{i} \phi_{k}\left(\lambda_{i}^{2}-\lambda_{0}^{2}\right)}}{\sum_{i=1}^{N} w_{i}},
$$

where $P_{i}=P\left(\lambda_{i}^{2}\right)$ for brevity, and $w_{i}$ is the weight of a data point. We refer to the companion paper for a formal derivation of these equations. The RMTF is given by

$$
R(\phi)=\frac{\sum_{i=1}^{N} w_{\mathrm{i}} \mathrm{e}^{-2 \mathrm{i} \phi\left(\lambda_{i}^{2}-\lambda_{0}^{2}\right)}}{\sum_{i=1}^{N} w_{i}}
$$

One is free to choose $\lambda_{0}$. We chose the wavelength corresponding to the weighted average $\lambda^{2}$, which minimizes the component of the transfer function orthogonal to the actual polarization vector at $\lambda_{0}$.

Figure 2 shows the RMTF corresponding to the sampling of $\lambda^{2}$ space that was obtained in this work. It shows the amplitude, the real part, and the imaginary part of the RMTF. The real part is the response parallel to the polarization vector at $\lambda_{0}$ and the imaginary part is the response orthogonal to it. The output of the RM synthesis procedure is a data cube with axes $\alpha, \delta$, and $\phi$. It has complex values $P=Q+\mathrm{i} U$.

\subsection{The RM-cube}

A total of 126 complex polarization images were used in the construction of the RM-cube. A range of Faraday depths from -300 to $+300 \mathrm{rad} \mathrm{m}^{-2}$ was synthesized. Beyond this range no features were detected. The recognition of real astronomical

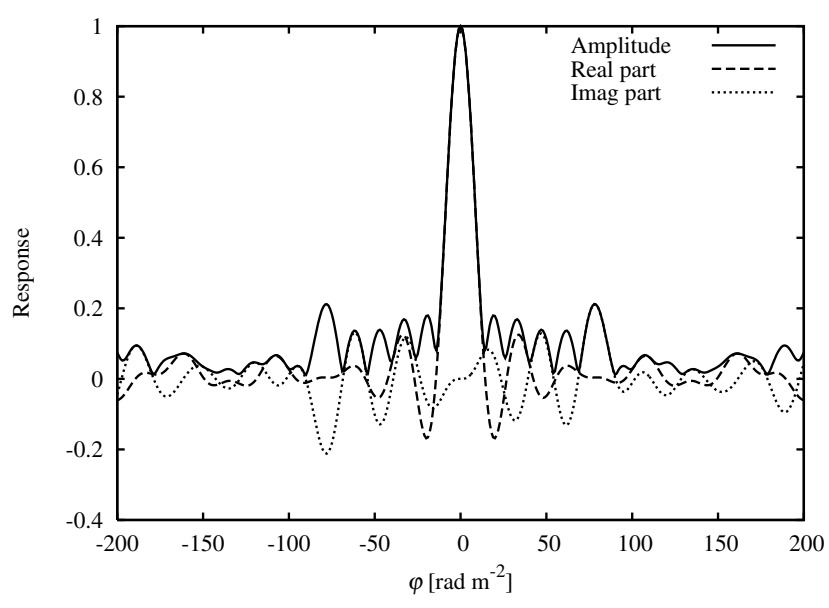

Fig. 2. The RMTF of the observations. The $F W H M$ is $15.2 \mathrm{rad} \mathrm{m}^{-2}$. The real part is the response parallel to the polarization vector and the imaginary part is the response orthogonal to it. Because of an uncertainty of the Faraday depth of the peak of typically a few $\mathrm{rad} \mathrm{m}^{-2}$ in low $\mathrm{S} / \mathrm{N}$ cases, no conclusions can be drawn with respect to the polarization angle at $\lambda=0$.

structures as well as instrumental artefacts is best done by animated scanning through the cube. Such a movie will be made available on our web page to help the reader in the recognition of the observed structures. A selection of frames from the RM-cube is shown in Fig. 7.

RM-synthesis adds polarization images over a considerable range of frequencies ranging from $315-370 \mathrm{MHz}$. These images would normally have angular resolutions that differ by a factor 1.2, or a factor 1.44 in beam area. In generating the half resolution image cube we therefore tapered to $1 / \mathrm{e}$ at a baseline of 1355 wavelengths, providing an almost frequency independent beam. In principle we could also correct the images for the different primary beam attenuations. However, this would cause a frequency dependent noise level which depends on radial distance. A full discussion of the various issues in dealing with primary beam attenuation, and the related issue of the intrinsic source emission spectrum, is presented in Brentjens \& de Bruyn (2005).

We have also made RM-cubes with full angular resolution, but we will only discuss in detail the half resolution images which have better $\mathrm{S} / \mathrm{N}$ for the generally extended emission. We have verified that this angular smoothing, which locally could lead to some beam depolarization, did not affect our conclusions in any significant way.

The addition of 126 complex images, each with a noise level of about $0.7-0.8 \mathrm{mJy}$, resulted in an RM-cube with rmsnoise levels going down to about $70 \mu \mathrm{Jy}_{\text {beam }}{ }^{-1}$ in Stokes $Q$ and $U$. However, these levels are only reached at the edge of the field and/or at high RM values $\left(>100 \mathrm{rad} \mathrm{m}^{-2}\right.$ ) where instrumental problems have been "wound up" sufficiently to be left only with the (uncorrelated) noise in the individual channels.

\section{Description of observed structures}

Figure 7 shows several representative frames from the total polarization RM-cube. Most of them are separated by about 
$9 \mathrm{rad} \mathrm{m}^{-2}$, which should be compared to the resolution in $\mathrm{RM}$-space of $15 \mathrm{rad} \mathrm{m}^{-2}$. These images have not been corrected for the reduced off-axis sensitivity in order to preserve a uniform noise level across the images. As discussed above, the images produced using the RM-synthesis technique utilize an effective bandwidth of about $50 \mathrm{MHz}$ yielding a sensitivity of better than $100 \mu \mathrm{Jy}_{\text {beam }}{ }^{-1}$. This is more than an order of magnitude below the total intensity noise level and with a peak brightness in Stokes $I$ of $22 \mathrm{Jy}$ (in the half resolution image) this represents a formal dynamic range of about $200000: 1$. It is clear, however, that the dynamic range varies enormously in the RM-cube. Thus in addition to the real (astronomical) signals from cluster synchrotron emission, the polarized images as well as the RM-cube images show many structures and patterns that have an instrumental origin. We will therefore begin with a description and, where known, an explanation of these instrumental artefacts. This will be followed by a description of the astronomical features.

\subsection{Instrumental artefacts}

We have identified several prominent patterns in the polarization and RM-cube images that clearly have an instrumental origin. In recognizing instrumental artefacts it is important to realize that they are a combination of uv-plane and image-plane effects. Multiplicative errors in the uv-plane result in a convolution with an error pattern in the image plane. The strongest effects are therefore associated with the strongest sources, although they can extend over a substantial part of the image plane. The off-axis effects, however, also have a multiplicative character resulting in a spatially dependent convolution with an error pattern. Again the strongest sources show the largest effects.

Radio emission observed away from the telescope pointing direction of reflector antennas is generally polarized. The component that is uniform across the field of view is small (a few \%) and is calibrated away using standard procedures (Sect. 3.2). The instrumental polarization (in Stokes parameters $Q, U$ and $V$ ) in parabolic dishes, however, rapidly increases with distance from the optical axis (Napier 1999). In the case of the WSRT it increases to about $20 \%$ at the $-10 \mathrm{~dB}$ power point with a typical clover-leaf pattern. Stokes $Q$ is positive in the $\mathrm{E}-\mathrm{W}$ direction and negative in the N-S direction. The Stokes $U$ pattern is rotated counterclockwise relative to Stokes $Q$ by $45^{\circ}$.

The off-axis polarization causes spurious signals at the location of the many strong sources in the field (see Fig. 4). The inevitable small spread in off-axis polarization levels for different telescopes in the array also causes weak ring-like patterns (see Fig. 4). Furthermore, the small variations between different $12 \mathrm{~h}$ observations also cause weak residuals at multiples of the grating lobes radius (about $40^{\prime}$, and frequency dependent). Intersection of these grating lobes then gives rise to a number of weak spurious features.

At $\mathrm{RM}=0$, all contributions add up coherently, thus if the off-axis polarization would be independent of frequency the response in the RM-cube should rapidly diminish at large $\mathrm{RM}$ values. The observed response as a function of RM indeed

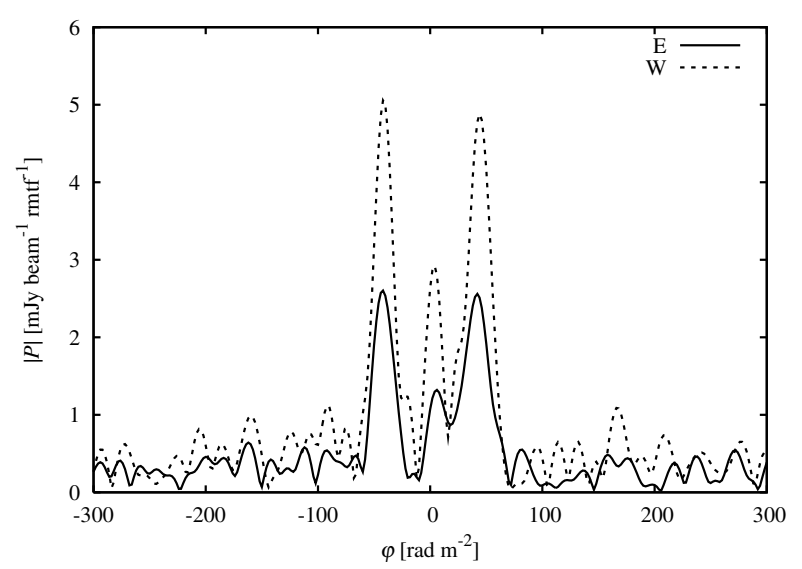

Fig. 3. Faraday spectrum at the location of the east (E) and west (W) lobe of the extended radio source at $\alpha \approx 3^{\mathrm{h}} 10^{\mathrm{m}}, \delta \approx 42^{\circ} 50^{\prime}$, revealing the instrumental polarization resonances for strong off-axis sources.

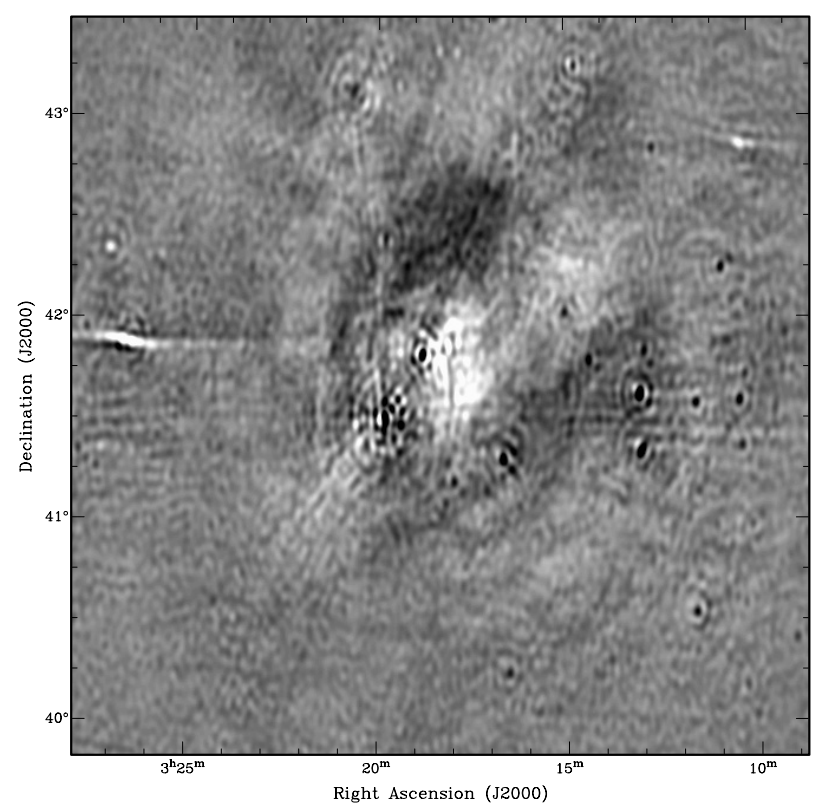

Fig. 4. Derotated Stokes $Q$ at $\lambda^{2}=0.759 \mathrm{~m}^{2}$ for the foreground emission at a Faraday depth $\phi=6 \mathrm{rad} \mathrm{m}^{-2}$. The intensity scale saturates at $+1.5 \mathrm{mJy} /$ beam (white) and $-1.5 \mathrm{mJy} /$ beam (black).

drops fairly rapidly but not as fast as expected. This is due to the fact that the off-axis polarization of the WSRT, in Stokes $U$ and to a lesser extent Stokes $Q$, also reveals a very strong frequency dependence with a period of about $17 \mathrm{MHz}^{2}$

The $17 \mathrm{MHz}$ period causes peaks in the RM "spectrum" at values of about $+42 \mathrm{rad} \mathrm{m}^{-2}$ and $-42 \mathrm{rad} \mathrm{m}^{-2}$, in addition to the emission at $0 \mathrm{rad} \mathrm{m}^{-2}$. These "resonances" can be clearly discerned in intense sources located far off-axis when scanning through the RM movie. The elongated source in the north-western upper corner of the image (Fig. 1) shows this very clearly (Fig. 3). Detailed scrutiny of the Faraday spectrum

2 This instrumental attribute has been known to exist for a long time but its origin is still not fully understood. It is believed that there is a component due to a standing wave pattern between the focus box and the dish (separated by $8.75 \mathrm{~m}$ ) and a component due to scattering off the four legged support structure of the focus box. 


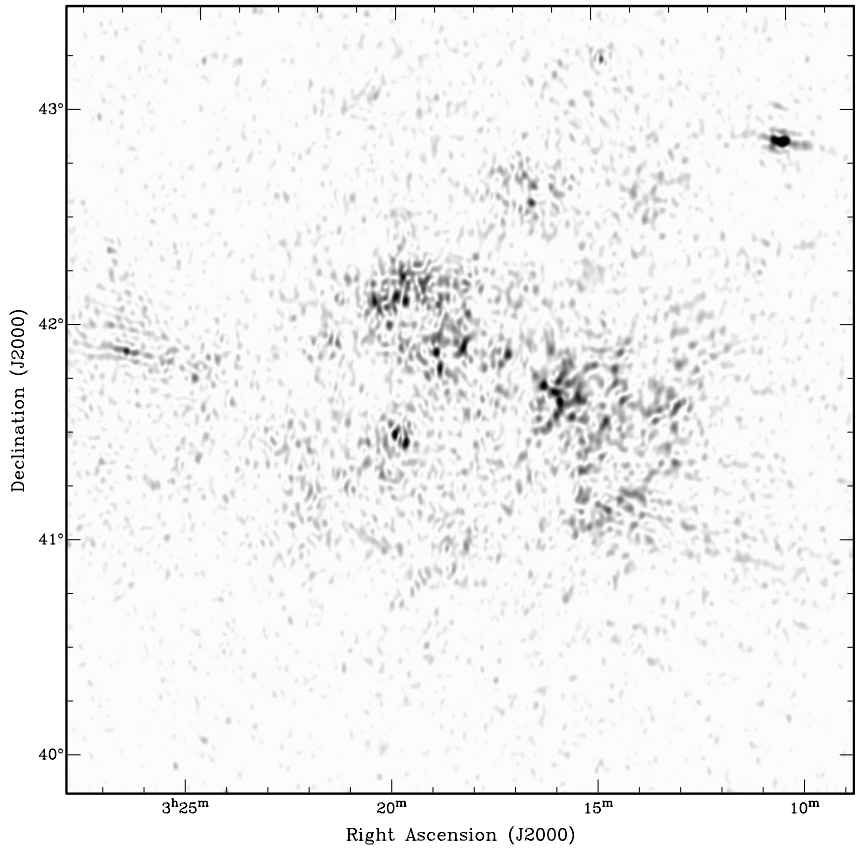

Fig. 5. Polarized intensity $\|P\|$ at $\phi=52 \mathrm{rad} \mathrm{m}^{-2}$. The grey scale covers a range from $0.3-2 \mathrm{mJy}_{\text {beam }}{ }^{-1} \mathrm{rmtf}^{-1}$.

over the face of the source suggests that the part of the emission around $\phi$ is $+5 \mathrm{rad} \mathrm{m}^{-2}$ may in fact be partly due to intrinsic polarization from the source. The predicted Galactic value at this location (see Sect. 7, Fig. 11) is about $-10 \mathrm{rad} \mathrm{m}^{-2}$.

A third instrumental artefact in the images is a faint pattern of radial stripes emanating from $3 \mathrm{C} 84$, by far the strongest radio source in the field. The intensity of these spikes rapidly dies out when we move away from position angles of $\pm 90^{\circ}$, giving them the appearance of "whiskers". Their peak intensity is about $0.5 \mathrm{mJy} \mathrm{beam}^{-1}$ but they are more typically present at $0.1-0.2$ mJy beam $^{-1}$. These whiskers are best seen in Fig. 5 but upon closer inspection they can also be seen in several of the frames of Fig. 7 (e.g. RM = -24, 21 and 51). Their origin is still being investigated. They were present only in the original Stokes $U$ (and $V$ ) images but the rotation of the polarization vector, after de-applying the ionospheric Faraday rotation, as well as the rotation inherent to the RM synthesis process itself, leads to their appearance in every RM frame.

Finally, we also detect polarized grating lobes from the source Cas A in the input $Q$ and $U$ image cubes. However, due to the great angular distance of this source from the field centre $\left(\approx 40^{\circ}\right)$ its chromatic grating response moves rapidly across the field of view as we move in frequency. In the final RM-cube, these instrumental features therefore decrease to a level well below the noise.

\subsection{Astronomical signals}

Once the instrumental features are recognized, the polarization images and the RM-cube frames are seen to be filled with highly significant signals of clearly celestial origin. They cover a wide range of Faraday depths $\phi$ between 0 and

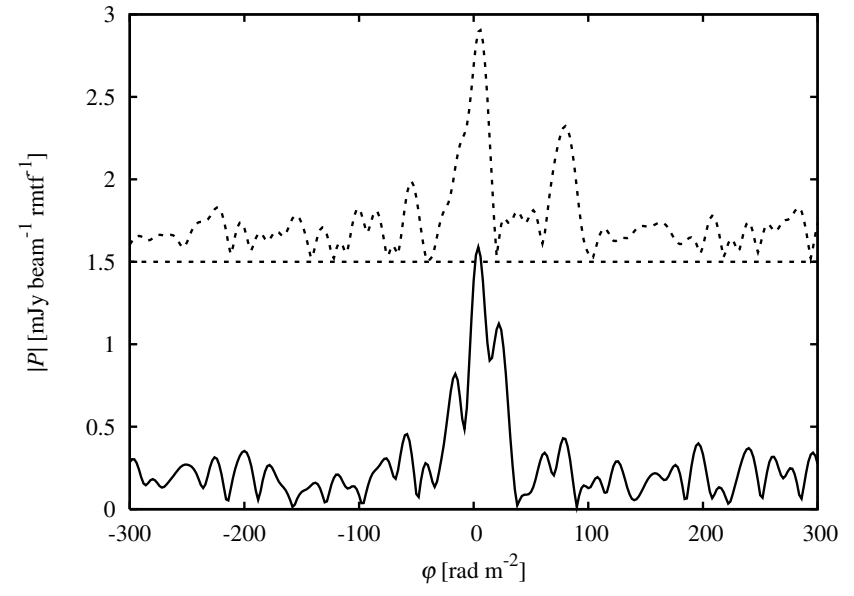

Fig. 6. Faraday depth spectra of two regions of Galactic foreground emission. The upper spectrum shows a region slightly west of the bar. The lower spectrum shows a foreground patch near the lens.

$90 \mathrm{rad} \mathrm{m}^{-2}$. Broadly speaking there seem to be two types of emission:

1. diffuse structures with slowly changing polarization angles on scales of the order of several tens of arc minutes: these have $0 \leq \phi \leq 15 \mathrm{rad} \mathrm{m}^{-2}$;

2. distinct large structures having sizes of the order of a degree and granularity in the polarization angle on scales of the order of a few arc minutes: these have $30 \leq \phi \leq 90 \mathrm{rad} \mathrm{m}^{-2}$.

There appears to be no significant emission between 15 and $30 \mathrm{rad} \mathrm{m}^{-2}$.

The first type of diffuse emission can be seen best in the third frame of Fig. 7. This diffuse emission appears to be spread over an area significantly wider than the primary beam $\left(2^{\circ} .2-2^{\circ} .6\right)$ although it does decrease in brightness, as any astronomical emission ought to. We emphasize this because the high Faraday depth emission is notably more confined to the central area. Figure 4 shows de-rotated Stokes $Q$ at a Faraday depth of $+6 \mathrm{rad} \mathrm{m}^{-2}$. It illustrates the large coherence of the polarization angle across these patches.

\section{Galactic foreground emission at low $\phi$}

The large-scale diffuse polarized emission at low $\phi$ is very similar to the features seen in previous WSRT studies at $92 \mathrm{~cm}$. This component has been shown to be produced in the relatively local Galactic medium, probably well within $1 \mathrm{kpc}$ from the Sun (Haverkorn et al. 2003a; Haverkorn 2002; Wieringa et al. 1993). This diffuse and complex polarization structure is believed to result from the line-of-sight superposition of intrinsically highly polarized emission and spatially as well as depth dependent Faraday rotation by the interstellar (Galactic) magneto-ionic medium. Haverkorn et al. (2003b) found that the rotation measure of this Galactic foreground in Auriga $(l=$ $\left.161^{\circ}, b=+16^{\circ}\right)$ is typically between -17 and $+10 \mathrm{rad} \mathrm{m}^{-2}$, with an average of $-3.4 \mathrm{rad} \mathrm{m}^{-2}$. The values we find towards the Perseus cluster at similar $l\left(l \approx 150^{\circ}\right)$ but opposite $b$ $\left(b \approx-13^{\circ}\right.$ ), are very similar. They cover the range from 0 to $12 \mathrm{rad} \mathrm{m}^{-2}$. 


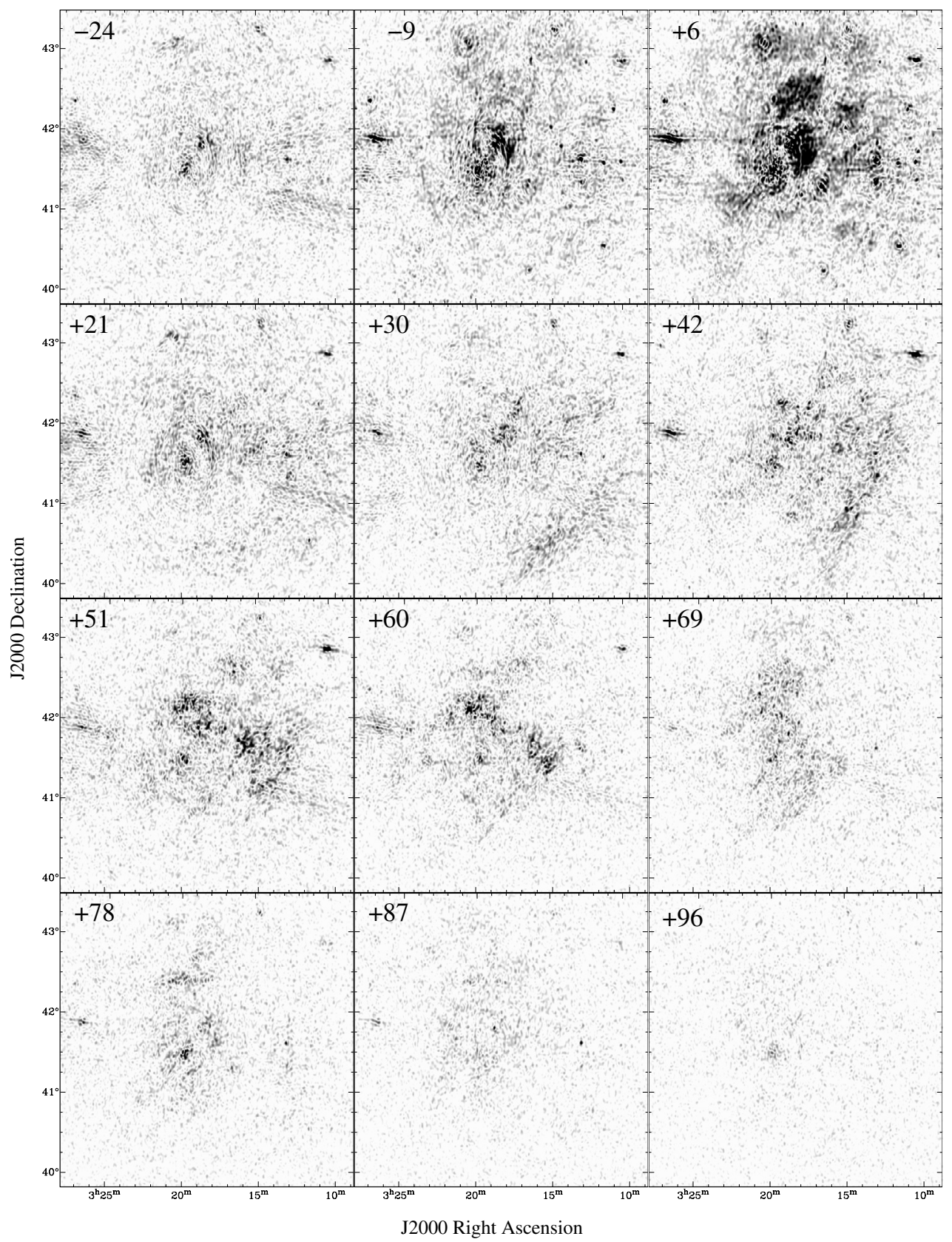

Fig. 7. Characteristic frames of polarized intensity $(\|P\|)$ from the RM-cube. The Faraday depth of each frame is specified in the top left corner in $\mathrm{rad} \mathrm{m}^{-2}$.

Figure 6 displays a few typical Faraday depth "spectra" of Galactic foreground patches, in areas where there is no significant emission due to (side lobes from) instrumentally polarized emission. It is also evident that at least part of the foreground is "extended" or "thick" in Faraday depth. Both spectra suggest that part of the positive contribution to the Faraday depth occurs inside the medium that produces the foreground emission. One should nevertheless not be misguided by the height of the peaks in a Faraday spectrum. The various artefacts in the individual $Q$ and $U$ images that were described above occasionally conspire to produce spurious peaks and one should therefore inspect the individual $Q$ and $U$ images at the location of a peak in a RM-movie to verify the reality of the RMfeature. Spatial continuity is a powerful aid in ascertaining the reality of most features, and this is best done in the RM-movie.

We find no exceptionally bright foreground features directly in front of $3 \mathrm{C} 84$ that could be associated with the low surface brightness polarized emission that was detected in very high dynamic range $21 \mathrm{~cm}$ WSRT observations (Sijbring 1993; de Bruyn 1995). We will return to this in Sect. 8.4.

\section{Background structures at high $\phi$}

\subsection{Description of structure in the RM-cube}

The second type of diffuse polarized emission is much richer in spatial structures. Note that we will only discuss the 


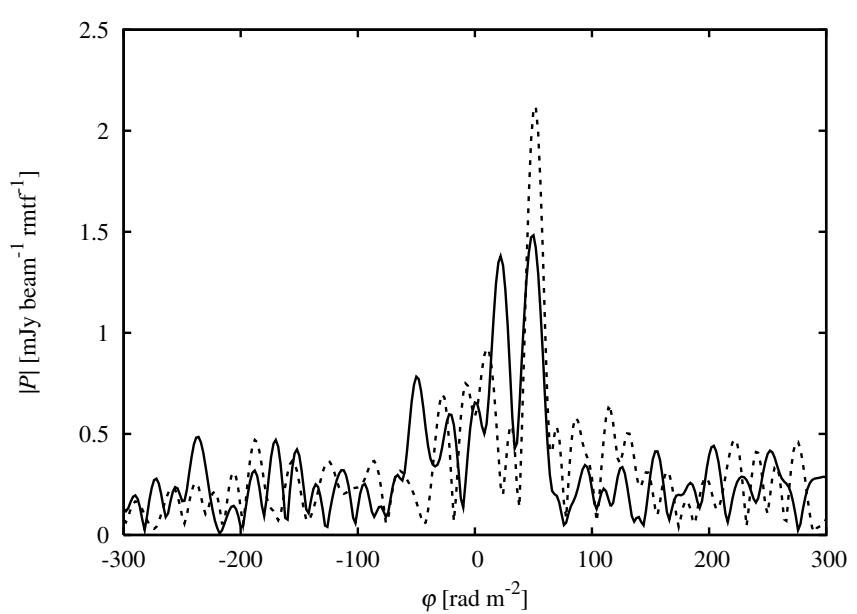

Fig. 8. Faraday depth spectra of two lines-of-sight through the doughnut.

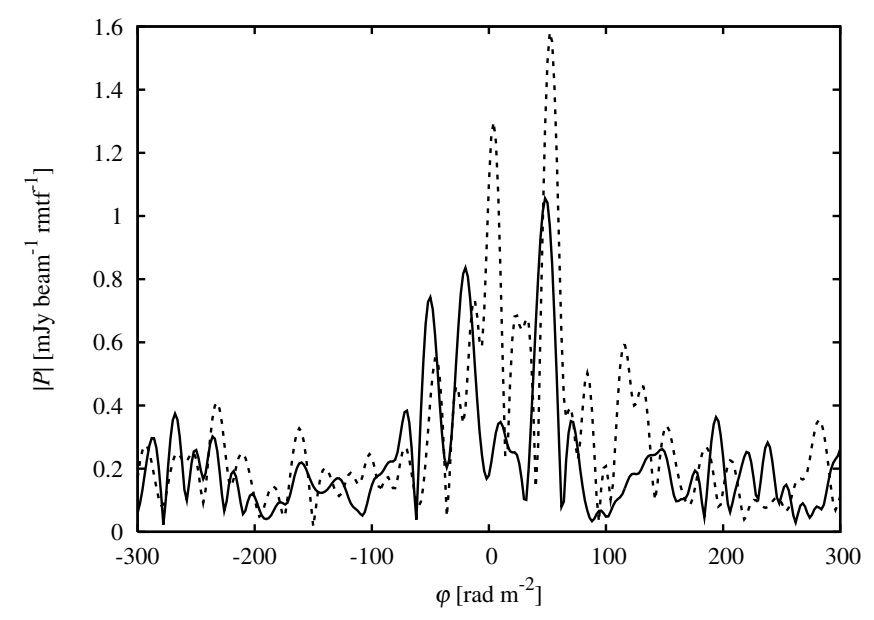

Fig. 9. Faraday depth spectra of two lines-of-sight through the lens.

polarized intensity and defer to a future paper the discussion and analysis of the rich structure in the polarization angle distribution. The description starts with the fifth frame of Fig. 7 $\left(\phi=30 \mathrm{rad} \mathrm{m}^{-2}\right.$ ) which shows a weak, front-like structure running from $\alpha \approx 3^{\mathrm{h}} 16^{\mathrm{m}}, \delta \approx 40^{\circ} 24^{\prime}$ to $\alpha \approx 3^{\mathrm{h}} 10^{\mathrm{m}}, \delta \approx 41^{\circ} 36^{\prime}$. The next frame $\left(\phi=42 \mathrm{rad} \mathrm{m}^{-2}\right)$ displays a stronger linear feature with a slightly different position angle. A bright circular "doughnut shaped" structure, with a diameter of about $7^{\prime}$ develops at position $\alpha \approx 3^{\mathrm{h}} 15^{\mathrm{m}} 35^{\mathrm{s}}, \delta \approx 41^{\circ} 42$ '. 3 . Figure 8 shows Faraday depth spectra of two lines-of-sight through the doughnut. The dashed spectrum shows the high significance of the doughnut. The peak at negative Faraday depth in the solid spectrum is caused by a whisker. The bright peak near $\phi=+10 \mathrm{rad} \mathrm{m}^{-2}$ is due to the Galactic foreground.

Frame number seven $\left(\phi=51 \mathrm{rad} \mathrm{m}^{-2}\right)$ shows a spectacular lenticular feature southwest of the doughnut. It is even better seen in Fig. 5, which is an enlarged frame from the RM-cube at $\phi=+52 \mathrm{rad} \mathrm{m}^{-2}$. The position angle of the lens is very similar to the position angle of the linear structure in the previous frame. The lens is roughly $40^{\prime} \times 20^{\prime}$ in size. At the distance of the Perseus cluster, it translates to $1 \times 0.5 \mathrm{Mpc}$. Two Faraday depth spectra through the lens are shown in Fig. 9.

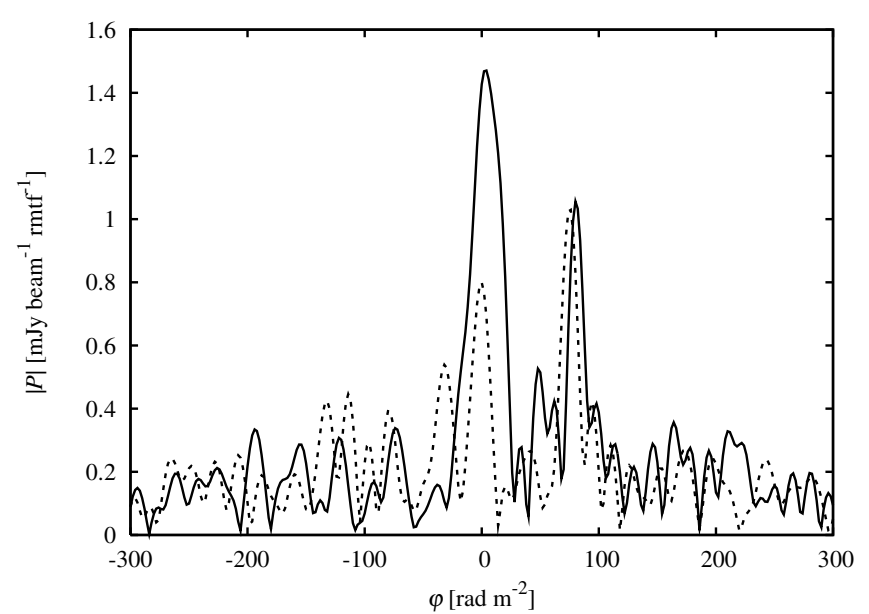

Fig. 10. Faraday depth spectra of two lines-of-sight through the straight bar north east of NGC 1265.

The two peaks at negative $\phi$ in the solid spectrum are caused by instrumental whiskers. The emission from the lens peaks at $\phi \approx 52 \mathrm{rad} \mathrm{m}^{-2}$. This frame and the next one, frame 8 at $\phi=60 \mathrm{rad} \mathrm{m}^{-2}$, also show bright extended emission with a polarized intensity of about $1 \mathrm{mJy}^{-1}$ beam $^{-1}$ approximately $40^{\prime}$ north of $3 \mathrm{C} 84$. This patch is roughly $15^{\prime} \times 25^{\prime}$ and is located north-west of the steep spectrum tail of NGC 1265 (Sijbring \& de Bruyn 1998).

The lens and doughnut slowly fade away in the frames that follow and polarized emission disappears from the western part of the field. Frame nine shows mottled emission across an area of $2^{\circ} \times 1.5$ centred around the area between NGC 1275 (3C 84) and NGC 1265. The tenth frame ( $\left.\phi=78 \mathrm{rad} \mathrm{m}^{-2}\right)$, is the last frame that shows significant emission. The horizontal bar almost $1^{\circ}$ due north of $3 \mathrm{C} 84$, at $\alpha \approx 3^{\mathrm{h}} 20^{\mathrm{m}}, \delta \approx 42^{\circ} 25^{\prime}$, is very conspicuous at these values of $\phi$. Two Faraday depth spectra are shown in Fig. 10. The emission fades away towards the cluster centre. Beyond $\phi \approx 100 \mathrm{rad} \mathrm{m}^{-2}$ no structures are detectable at $2^{\prime}-3^{\prime}$ resolution.

\subsection{Where is the total intensity counterpart!?}

We have not yet detected any of the extended polarized features in our RM-cube of the total intensity image. This was rather surprising because we have no doubt that this emission is due to the synchrotron process. There may, however, be a rather simple explanation. The sensitivity in the Stokes $I \mathrm{im}$ age is about a factor $10-15$ poorer than in the polarization image: $1.5 \mathrm{mJy}$ beam $^{-1}$ as against $0.1 \mathrm{mJy}$ beam $^{-1}$ (both numbers now refer to the $2^{\prime} \times 3^{\prime}$ resolution image. However, the brightest features in the polarization images, e.g. the "lens" and the "doughnut", have a peak brightness of about $1 \mathrm{mJy}^{\text {beam }}{ }^{-1}$. So the lack of detection in $I$ implies, at face value, a polarization percentage of at least 50\%. Although this is not impossible for synchrotron emission, these percentages are getting "uncomfortably" high. Several "relic" structures in clusters of galaxies (Enßlin et al. 1998) have polarization percentages of $20-30 \%$ although these all refer to shorter wavelengths where depolarization is less of an issue. 


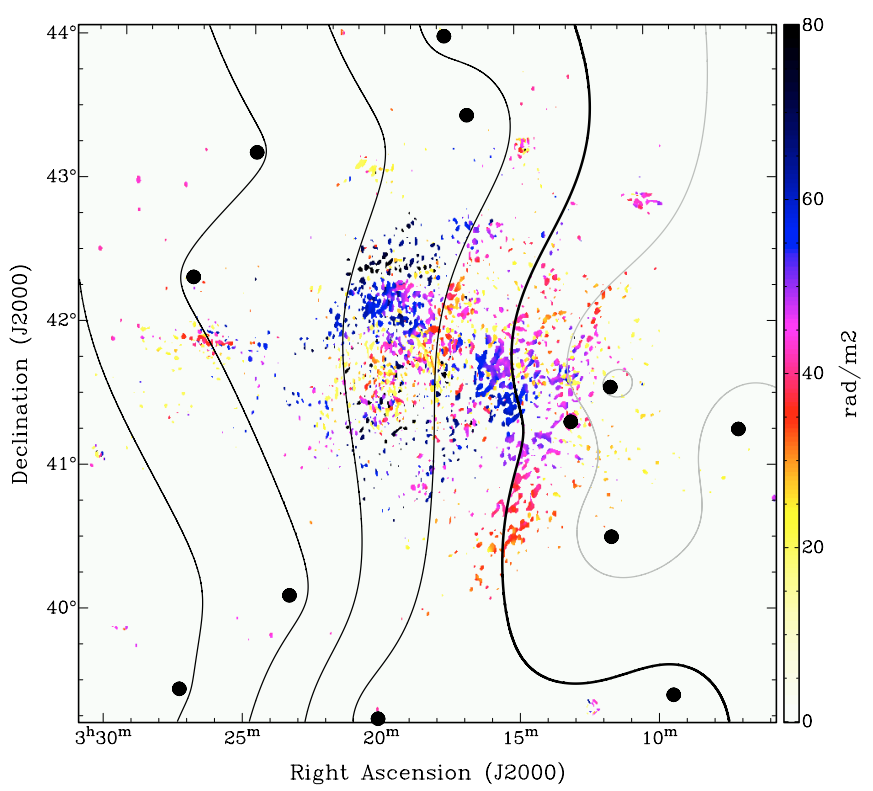

Fig. 11. Faraday depth of the maximum $|P|$ along the line-of-sight, excluding range 3-19 inclusive. Pixels with a peak polarized flux density less than $0.7 \mathrm{mJy}$ per beam have been masked out. Overplotted is a radial basis function interpolation (Carr et al. 2001) of the "foreground RM" determined using the 12 sources indicated with filled circles. The thick line has a $\mathrm{RM}=0 \mathrm{rad} \mathrm{m}^{-2}$, and contours are given in steps of $20 \mathrm{rad} \mathrm{m}^{-2}$ with positive values increasing to the east.

Apparent polarization percentages well above $100 \%$ are quite normal in the case of Galactic foreground polarization (Wieringa et al. 1993; Haverkorn et al. 2003a; Haverkorn 2002; Gaensler et al. 2001). They are due to the fact that the Galactic foreground Stokes $I$ is very smooth and largely resolved out due to the lack of sufficiently short interferometer spacings. The emission in Stokes $Q$ and $U$, on the other hand, has high spatial frequencies because they can be both positive and negative (cf. Fig. 4). Figure 11, to which we will return later, shows that the distribution of polarized intensity indeed has a significant large-scale component. If the total and polarized intensity would be distributed similarly, the shortest spacings of the WSRT will have only partly sampled the signals. This was exacerbated by the fact that in two out of six usable frequency bands one of the crucial telescopes (RT9 and RTA) providing the shortest $36 \mathrm{~m}$ spacing were not functioning properly. The true polarization percentage of the polarization structures could then easily be a factor 2-3 lower which would decrease the apparent percentage polarization from $>50 \%$ to $>20 \%$. The conclusion, however, remains that the signals must be highly polarized. We will return to this aspect below.

\section{Discussion}

\subsection{Location of high Faraday depth features}

There are two possible locations for the source of the emission at $\phi \geq 30 \mathrm{rad} \mathrm{m}^{-2}$. The first possibility is that here too we are dealing with Galactic synchrotron emission. Potential sites are the region between us, and possibly including, the Perseus arm which is at a distance of $2-3 \mathrm{kpc}$. The second possible location is the Perseus cluster of galaxies, which was the central target of these observations. We favour the second option for a number of reasons:

- the high $\phi$ structures seem to be largely confined to and approximately centred at the Perseus cluster, contrary to the low $\phi$ emission;

- there is a systematic decrease in the small scale structure in Stokes $Q$ and $U$ as a function of distance to the cluster centre.

Initially we believed there was a third argument for an association with the Perseus cluster: when scanning the RM-movie there is a very suggestive systematic convergence of the higher RM values towards the centre of the Perseus cluster. However, we could not exclude that this was caused in part by an anomaly in the Galactic foreground RM distribution.

Johnston-Hollitt et al. (2004) have constructed an interpolated map of the RM distribution of a sample of over 800 radio sources, from which we may estimate the Galactic RM contribution in any direction. The expected RM near the Perseus cluster is somewhere between +10 and $+20 \mathrm{rad} \mathrm{m}^{-2}$. The RM expected from the large-scale Galactic field should be negative in this area. The positive values inferred by Johnston-Hollitt et al. (2004) may therefore indicate an RM anomaly south of the Galactic Perseus arm.

\subsection{Evidence for a Galactic RM gradient}

To investigate this further and put our suspicions about foreground contamination to the test we proposed a new series of observations in August 2004, when most of this work had already been finished and written up. Using the WSRT at $21 \mathrm{~cm}$ we conducted a series of snapshot observations of 15 polarized (background) radio sources in the direction of the Perseus cluster. The sources were selected through the NVSS (Condon et al. 1998) and should have at least a few mJy of polarized emission. Using the $160 \mathrm{MHz}$ wide WSRT band this appeared to be sufficient to allow a RM determination accurate to better than a few rad m $\mathrm{m}^{-2}$ for all 15 sources after $30 \mathrm{~m}$ of integration per source. After excluding three sources with a complicated brightness morphology and multiple, widely different RMs, the remaining 12 sources showed a smooth gradient across the field of view. The results are shown in Fig. 11 and include a radial basis-function interpolation of a smooth foreground screen (Carr et al. 2001). Because the uncertainty in the RM determination of each source is at most a few $\mathrm{rad} \mathrm{m}^{-2}$, the gradient is very significant. We also infer that the scatter due to a RM contribution from the medium inside, or surrounding, the radio sources can be at most $5-10 \mathrm{rad} \mathrm{m}^{-2}$; this is consistent with previous studies of RM differences in double radio sources (Simonetti et al. 1984; Simonetti \& Cordes 1986; Leahy 1987).

This smooth Galactic foreground RM gradient should be compared with the observed spatial distribution in the diffuse high Faraday depth emission features. To do this we proceeded as follows but we begin with noting that there is no such thing as the RM or the Faraday depth of a pixel: indeed there can be multiple regions of emission at different Faraday depths along the line-of-sight (for an extensive discussion of such 
situations, which are quite normal at low frequencies, we refer to Brentjens \& de Bruyn 2005). Within the RM-cube we masked out the emission at $3 \leq \phi \leq 19 \mathrm{rad} \mathrm{m}^{-2}$ which is very clearly dominated by the Galactic foreground. At every pixel in the RM-cube we then determined at which Faraday depth the polarized intensity $|P|$ was maximal. This range included $\phi=0 \mathrm{rad} \mathrm{m}^{-2}$, the value where all frequency independent instrumental problems accumulate. This guaranteed that the emission that would be assigned a Faraday depth other than zero, cannot be caused by RMTF side lobes of instrumental problems. The regions where the latter is manifestly the case are the sources at the edges of the field. The result of this exercise is shown in Fig. $11^{3}$. We imposed a cut at $|P|<0.7 \mathrm{mJy}^{-1}$ beam $^{-1}$ before including pixels.

Figure 11 reveals that across the $2^{\circ}$ diameter area with high $\phi$ emission there is a clear Galactic RM gradient of about $60 \mathrm{rad} \mathrm{m}^{-2}$, which is approximately equal to the observed range of $\phi=30-90 \mathrm{rad} \mathrm{m}^{-2}$. In other words, the high $\phi$ emission shows a fairly uniform positive excess of about $40-50 \mathrm{rad} \mathrm{m}^{-2}$ relative to the interpolated Galactic RM. For all but two of the background sources the line-of-sight avoids the Perseus cluster, which we define as the area out to which significant X-ray emission can be seen, a radius of about $1^{\circ}$. We may therefore assume that the RM of these background radio sources represents the line-of sight-integrated RM of our Galactic foreground magneto-ionic medium. This result holds the key to the interpretation of the high $\phi$ emission as we will now proceed to argue.

For the sake of the argument let us concentrate on the features on the western side of the cluster near what we have called the shock, the lens and the doughnut. The diffuse organized Galactic polarized emission has a RM of about $+10 \mathrm{rad} \mathrm{m}^{-2}$. In order for the total, integrated, RM to the background sources to end up at about $0 \mathrm{rad} \mathrm{m}^{-2}$ there must be another small negative contribution of about $-10 \mathrm{rad} \mathrm{m}^{-2}$ from somewhere along the line-of-sight in our Galaxy. This seems not implausible. The RM-cube around $\phi \approx 0 \mathrm{rad} \mathrm{m}^{-2}$ is rather messy due to the remaining instrumental polarization from the extremely bright and complex cluster radio sources. Now let us suppose that the "screen" responsible for the $40-50 \mathrm{rad} \mathrm{m}^{-2}$ contribution is also due to our Galaxy and extends across the full field of view shown in Fig. 11 (the sudden termination at a distance of about 1.5 from the pointing centre must then be attributed largely to the primary beam attenuation, which is unlikely but not impossible). We would then require another screen, but now with a Faraday depth of $\phi=-40 \mathrm{rad} \mathrm{m}^{-2}$ to compensate for the $\phi=+40 \mathrm{rad} \mathrm{m}^{-2}$ screen. We see no evidence in our RM-cube for any emission from such a screen.

Even stronger arguments can be brought forward for the case that the $\phi=+40 \mathrm{rad} \mathrm{m}^{-2}$ emission would result from a discrete cloud in the Perseus arm of our Galaxy that just happened to cover the Perseus cluster. Assuming a magnetic field of $1 \mu \mathrm{Gauss}$, a depth of about 60 parsec (equal to a lateral scale of 1.5 at $2.5 \mathrm{kpc}$ ), a $\mathrm{RM}$ of $40 \mathrm{rad} \mathrm{m}^{-2}$ requires a (smooth) electron density of $0.8 \mathrm{~cm}^{-3}$. Assuming roughly $20 \% \mathrm{H} \alpha$

\footnotetext{
${ }^{3}$ The on-line version of this article contains a colour version of this figure.
}

extinction, this density and path length would generate an $\mathrm{H} \alpha$ surface brightness of about 13 Rayleighs. The total $\mathrm{H} \alpha$ surface brightness towards the Perseus cluster in the WHAM survey (Haffner et al. 2003) is about 4 Rayleighs. Furthermore, the integrated $\mathrm{H} \alpha$ emission is rather smooth towards the Perseus cluster, ranging in surface brightness from 3-5 Rayleighs over an area of $3^{\circ} \times 3^{\circ}$ centred on the Perseus cluster. There is no excess at the location of the cluster. The discrete Perseus arm model for the origin of the excess $+40 \mathrm{rad} \mathrm{m}^{-2}$ polarized emission therefore appears to be untenable as well.

A similar argument can be given for the emission to the north and east of the cluster centre. The emission in this region shows a significant drop in surface brightness well before the primary beam attenuation sets in. This would have to be a peculiar coincidence in the case of a Galactic foreground origin.

When we add the above arguments to those given previously, the spatial coincidence with the cluster and the granularity of the polarization angle structure, we are led to the conclusion that the high $\phi$ emission indeed must be associated with the Perseus cluster of galaxies.

\subsection{Intracluster or peripheral emission?}

Having argued that the high $\phi$ emission is associated with the Perseus cluster we may then ask the question: where in the cluster does the emission originate? Does it originate from within the cluster or is it emitted from the periphery of the cluster? At this point we should also realize that this question really has two aspects:

1. Where does the emission come from?

2. Where does the Faraday rotation occur?

Figure 12 is a sketch that is helpful for the following discussion. It shows where we believe the emission and rotation occur in the Perseus cluster. From the fact that most of the polarized structures are unresolved by the RMTF it appears that the medium is not Faraday-thick (see Brentjens \& de Bruyn 2005). This means that the largest fraction of the Faraday rotation of $40 \mathrm{rad} \mathrm{m}^{-2}$ occurs between the emitting region and us.

At this point it is relevant to recall that there are two extended and polarized radio sources within the Perseus cluster, NGC 1265 and IC 310, in the area where we find diffuse polarized emission. Their head-tail morphology indicates that they are most likely located within the denser gaseous part of the Perseus cluster (Miley et al. 1972). The RM across the bright part of the tail of NGC 1265 (O'Dea \& Owen 1986, 1987) shows a scatter of $20 \mathrm{rad} \mathrm{m}^{-2}$ around a mean value of $45-50 \mathrm{rad} \mathrm{m}^{-2}$. The scatter is probably due to the magnetized plasma in the cocoon surrounding and mixed within the twin tails of this head-tail source. The "expected" value due to our Galactic foreground at the location of NGC 1265 is about $25 \mathrm{rad} \mathrm{m}^{-2}$ (cf. Fig. 11). The difference of about $20 \mathrm{rad} \mathrm{m}^{-2}$ must then be attributed to the medium in the line-of-sight between the head-tail source and the edge of our Galaxy: i.e. the peripheral region of the near side of the cluster.

We also detect faint but highly significant polarized emission from a region close to the "head" of IC 310 at a value of $\phi=+80 \mathrm{rad} \mathrm{m}^{-2}$ (Fig. 16). This polarized emission probably 
Faraday rotation

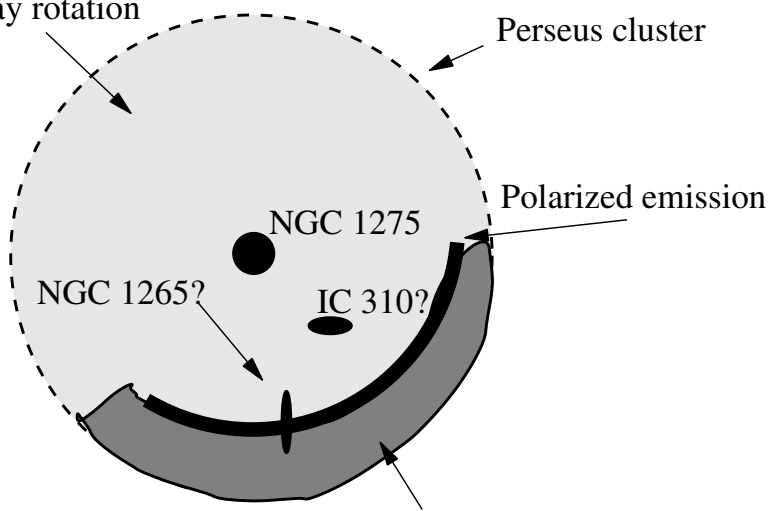

Faraday rotation $\left(+40 \mathrm{rad} \mathrm{m}^{-2}\right)$

Earth

Fig. 12. Sketch of our proposal for the situation in the Perseus cluster. A top view of the distribution in the Perseus cluster of various emitting areas and Faraday rotating areas.

originates from a region about $1^{\prime}$ downstream from the head of IC 310 where significant polarized emission was also detected at $610 \mathrm{MHz}$ by Sijbring \& de Bruyn (1998). This is sufficiently far away from the galaxy that we may assume that the Faraday rotation is not due to the ISM associated with IC 310 itself. The expected Galactic RM value (cf. Fig. 11) at this location is about $+20 \mathrm{rad} \mathrm{m}^{-2}$. The difference of $+60 \mathrm{rad} \mathrm{m}^{-2}$ could imply that IC 310 is located deep within the cluster.

Note that the excess Faraday depth shown by NGC 1265 and IC 310 is consistent with the average cluster RM excess of less than $50 \mathrm{rad} \mathrm{m}^{-2}$ found by Clarke et al. (2001) at the projected distance of $700 \mathrm{kpc}$ of these two head-tail radio sources from the centre of the Perseus cluster. The polarized emission having $\phi=40 \mathrm{rad} \mathrm{m}^{-2}$ is probably located on the periphery on the near side of the cluster at about the same depth as NGC 1265. IC 310 may well be located behind the diffuse polarized emission.

\subsection{Thomson scattering within the Perseus cluster?}

As discussed in the introduction the initial goal of the observations was to characterize the possible contamination of Galactic foreground polarization to the observed $21 \mathrm{~cm}$ polarization (de Bruyn 1995) which was observed to straddle 3C 84 on an angular scale of $30^{\prime}$ and believed to be due to Thomson scattering. In a future paper we plan to present the original $21 \mathrm{~cm}$ observations augmented with a wider field study.

Could the polarized emission that we detected at wavelengths of 81-95 $\mathrm{cm}$ be indeed Thomson scattered radiation from 3C 84 or its direct surroundings (Syunyaev 1982; Wise \& Sarazin 1990, 1992) ${ }^{4}$ ?

${ }^{4}$ Please note that Wise \& Sarazin (1990) made a small error in their Eq. (1), which makes it incompatible with our Eqs. (10) and (11). Equation (1) of Wise \& Sarazin (1990) must be multiplied by $1 / 4 \pi$ in order to correct this. The same error is also present in Eq. (2.1) of Wise \& Sarazin (1992), hence their estimates are one order of magnitude too optimistic.

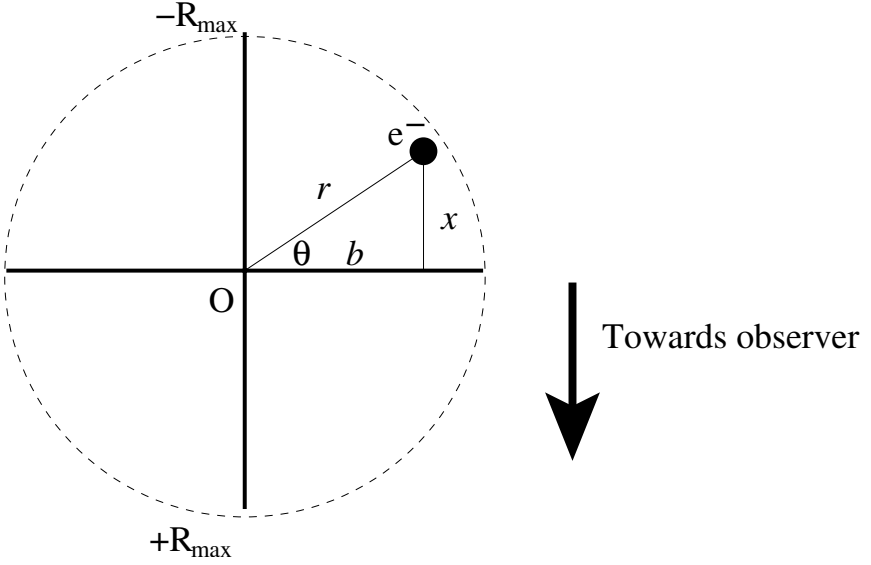

Fig. 13. Thomson scattering geometry.

In order to quantitatively answer that question, we have made a simple model of the Perseus cluster. The redshift of the Perseus cluster is $z=0.0167$ (Struble \& Rood 1999). We assume $H_{0}=72 \pm 2 \mathrm{~km} \mathrm{~s}^{-1} \mathrm{Mpc}^{-1}$ (Spergel et al. 2003) which gives a distance to the cluster of $69.5 \pm 2 \mathrm{Mpc}$. We have adopted a spherically symmetric electron distribution. We used the deprojected radial dependence determined by Churazov et al. (2003):

$n_{\mathrm{e}}=\frac{3.9 \times 10^{-2}}{\left[1+\left(\frac{r}{80 \mathrm{kpc}}\right)^{2}\right]^{1.8}}+\frac{4.05 \times 10^{-3}}{\left[1+\left(\frac{r}{280 \mathrm{kpc}}\right)^{2}\right]^{0.87}} \mathrm{~cm}^{-3}$.

In order to estimate the radio radiation, we summed the contributions of the $3 \mathrm{C} 8430^{\prime \prime}$ component and the halo. We ignore the very bright core, because it has an uncertain history and its high flux density is largely due to an outburst that only started in the late 1950's (O'Dea et al. 1984). Its Thomson echo cannot therefore have spread by more than a few tens of parsecs. The values for the luminosity from the extended components are taken from Sijbring (1993) after scaling them to our adopted distance. Both source components were situated in the centre of gravity of the electron population. We modelled the source as a single unpolarized point source and assumed an isotropic radiation field.

$$
\begin{aligned}
P\left(v^{\prime}\right)= & 10.2 \times 10^{24}\left(\frac{v^{\prime}}{333 \mathrm{MHz}}\right)^{-1.15} \\
& +9.83 \times 10^{24}\left(\frac{v^{\prime}}{333 \mathrm{MHz}}\right)^{-1.2} \mathrm{~W} \mathrm{~Hz}^{-1},
\end{aligned}
$$

where $v^{\prime}=(1+z) v$ is the rest frame frequency of the Perseus cluster.

The geometry of the computation is given in Fig. 13. The radio source is located at the origin $(\mathrm{O})$. The emission is scattered at location $(b, x)$. The total intensity of the scattered emission is given by the line integral

$I(v)=\int_{-\infty}^{+\infty} \frac{P((1+z) v)}{4 \pi r^{2}} \frac{\mathrm{d} \sigma}{\mathrm{d} \Omega} n_{\mathrm{e}}(\boldsymbol{r}(x, b)) \mathrm{d} x$,

where

$\frac{\mathrm{d} \sigma}{\mathrm{d} \Omega}=\frac{1}{2} r_{0}^{2}\left(1+\sin ^{2} \theta\right)$ 
and

$r_{0}^{2}=\frac{3}{8 \pi} \sigma_{\mathrm{T}}$.

(Syunyaev 1982; Rybicki \& Lightman 1979). We can write Eq. (7) in terms of $b$ and $x$ as depicted in Fig. 13.

$I(v)=K(v) \int_{-L}^{+L} n_{\mathrm{e}}(r) \frac{b^{2}+2 x^{2}}{\left(b^{2}+x^{2}\right)^{2}} \mathrm{~d} x \mathrm{~W} \mathrm{~Hz}^{-1} \mathrm{~m}^{-2} \mathrm{Sr}^{-1}$,

where $b$ is the impact parameter, $x$ is the path length measured from the midplane of the electron distribution, $r=\sqrt{b^{2}+x^{2}}$ is the distance from the radio source, $L=\sqrt{R_{\max }^{2}-b^{2}}$ is the integration boundary, and

$K(v)=\frac{3 P((1+z) v) \sigma_{\mathrm{T}}}{64 \pi^{2}(1+z)^{3}} \mathrm{~W} \mathrm{~m}^{2} \mathrm{~Hz}^{-1} \mathrm{Sr}^{-1}$,

independent of position. We used SI units throughout this computation. Assuming there is no Faraday rotation in the cluster medium, which of course is unrealistic at a wavelength of $92 \mathrm{~cm}$, we may also compute the maximum degree of polarization by

$I_{\mathrm{P}}(v)=K(v) \int_{-L}^{+L} n_{\mathrm{e}}(r) \frac{b^{2}}{\left(b^{2}+x^{2}\right)^{2}} \mathrm{~d} x \mathrm{~W} \mathrm{~Hz}^{-1} \mathrm{~m}^{-2} \mathrm{Sr}^{-1}$.

The results are shown in Figs. 14 and 15. Figure 14 indicates that at small projected distances $\left(<30^{\prime}\right)$ from the cluster core it is, in principle, possible to detect the Thomson scattering emission even at $92 \mathrm{~cm}$ wavelength. For example, at $25^{\prime}$ distance from the cluster centre, the expected total intensity is approximately $50 \mu \mathrm{Jy} 2^{\prime} \times 3^{\prime}$ beam. Assuming $50 \%$ polarization, this amounts to a polarized intensity of $25 \mu \mathrm{Jy}_{\text {beam }}{ }^{-1}$. Barring dynamic range issues, it will however be very difficult to separate this scattered emission from true cluster halo emission in Stokes $I$. The much deeper sensitivity reached in polarized intensity $\left(70 \mu \mathrm{Jy}_{\mathrm{beam}^{-1}}\right)$ affords a better chance if we can correctly estimate the complications from Faraday rotation within the scattering medium. So although we can not exclude that part of the mottled polarized emission close to $3 \mathrm{C} 84$, at the location where we previously observed significant polarized emission at $21 \mathrm{~cm}$, is due to Thomson scattering, we will not discuss it here any further and will concentrate on the brighter polarized features.

We believe that neither the large structures that we detected in the western part of the field, nor the bar feature to the north, can be explained by Thomson scattering. The high percentage of polarization inferred for the diffuse structures are consistent with Thomson scattering (without significant de-polarization), but they appear far too bright for their projected distance from $3 \mathrm{C} 84$. For example, to explain the observed polarized surface brightness of the lens (approximately $1 \mathrm{mJy}$ per $2^{\prime} \times 3^{\prime}$ beam) by Thomson scattering, either the source luminosity 3 to 6 million years ago should have been a factor of 100 to 500 higher, or the electron density at 1 to $2 \mathrm{Mpc}$ distance from the cluster centre should be at least $5 \times 10^{-3} \mathrm{~cm}^{-3}$. Both options, or a combination of a higher luminosity and a higher density, appear to be implausible. However, we note that the current core luminosity is $5 \times$ higher than the luminosity assumed in the computations and the uncertainty about the long term average of

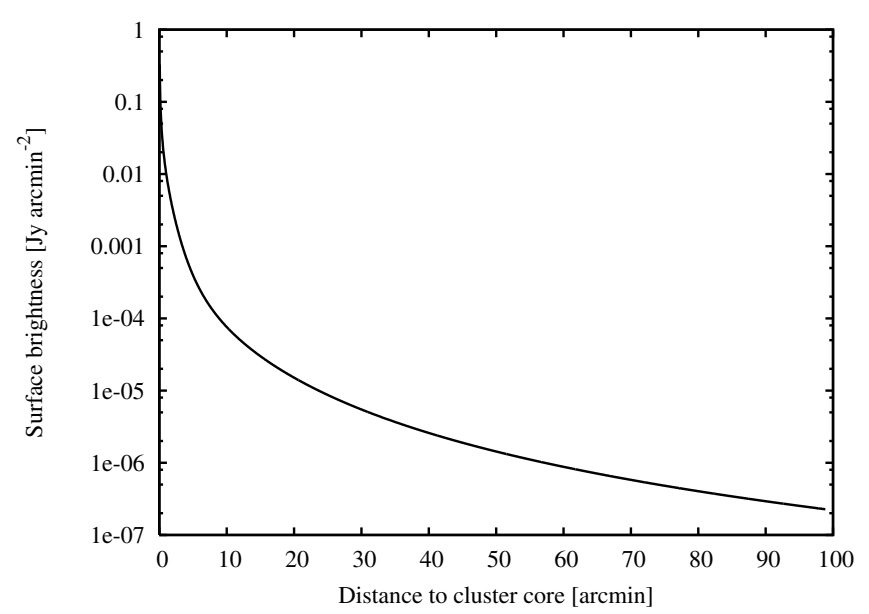

Fig. 14. Computed Thomson scattering halo surface brightness at $92 \mathrm{~cm}$ based on the radio flux density of the $3 \mathrm{C} 84$ halo and $30^{\prime \prime} \mathrm{com}-$ ponent. Total intensity.

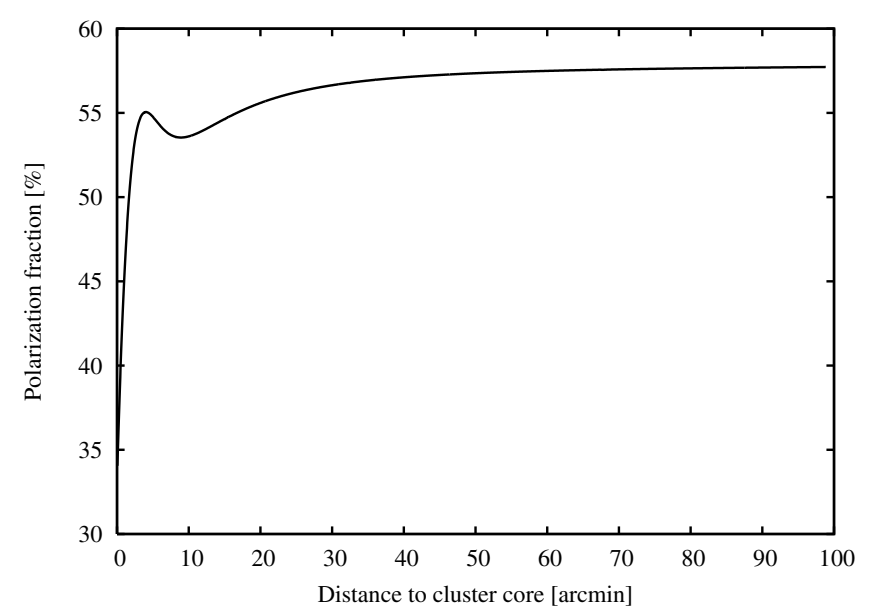

Fig. 15. Computed Thomson scattering halo surface brightness at $92 \mathrm{~cm}$ based on the radio flux density of the 3C 84 halo and $30^{\prime \prime}$ component. Polarization fraction.

the core emission will remain one of the uncertain issues with the Thomson scattering interpretation. In a future paper we will return to the issue of the separation of Thomson scattered emission from other Perseus cluster contributions using both existing and new $21 \mathrm{~cm}, 92 \mathrm{~cm}$, and $200 \mathrm{~cm}$ WSRT observations.

\subsection{Structure formation shocks and AGN bubbles}

We will now briefly discuss the implications of what we consider to be the most likely explanation for the nature of the diffuse high Faraday depth structures centred on the Perseus cluster. A more in-depth study will be presented in future papers. We will follow Enßlin et al. (1998) and Enßlin \& Gopal-Krishna (2001) and interpret the emission as resulting from (re-energized) relics and emission associated with shocks in the large-scale structure formation gas flow.

Cosmological simulations of structure formation have improved dramatically in recent years. Nearly all simulations indicate that structure in the Universe formed hierarchically. 


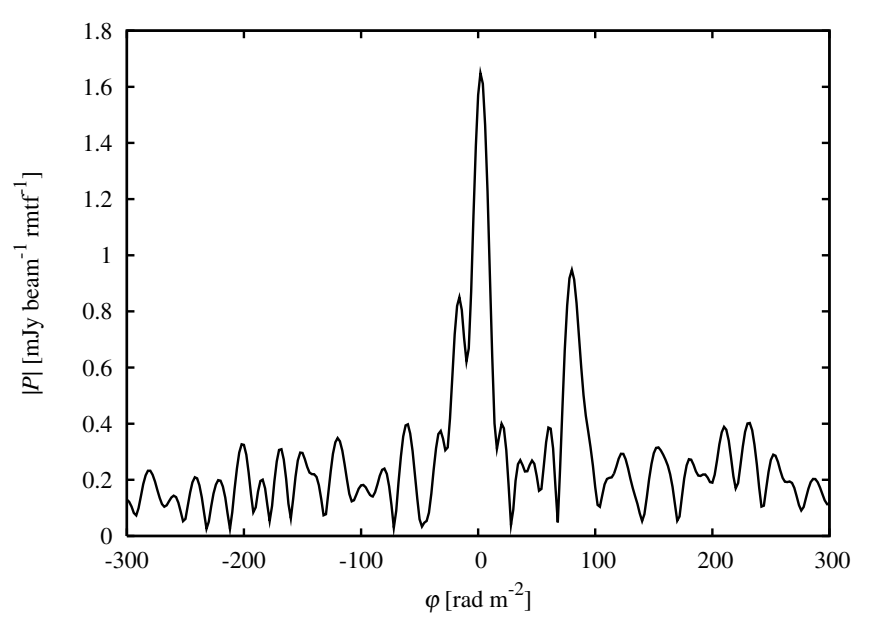

Fig. 16. Faraday depth spectrum near the "head" of IC 310. A highly significant feature at Faraday depth $\phi=80 \mathrm{rad} \mathrm{m}^{-2}$ can be detected. The peak around $\phi=0 \mathrm{rad} \mathrm{m}^{-2}$ is due to the instrumental polarization of IC 310, which has a peak in total intensity of more than $500 \mathrm{mJy}$.

Small clumps collapsed, larger ones started accreting smaller ones and grew even larger. A consequence of this type of structure formation is the presence of large-scale gas flows, which form huge, multi megaparsec scale shocks at their intersections (Burns 1998; Quilis et al. 1998; Miniati et al. 2000). Enßlin et al. (1998) discuss the possibility that cluster relic sources, large highly polarized radio sources in the outskirts of galaxy clusters, could trace interface shocks between clusters and the super cluster filaments accreting onto them. The electrons in the relic would be re-energized by diffusive shock acceleration. Enßlin \& Gopal-Krishna (2001) developed an analytic formalism for an alternative process. They propose that cluster relics are bubbles of magnetized plasma, released into the IGM by AGN. The electrons in such a bubble loose energy through adiabatic expansion of the bubble. Once fully detached, the bubble enters a "hibernation" phase in which it can stay for several Gyr; the only energy losses suffered by the relativistic electrons are those due to Inverse Compton losses off the $2.7 \mathrm{~K}$ background radiation. The bubble is now buoyant in the IGM, due to its lower density (Brüggen 2003). It is finally revived when the bubble is adiabatically compressed by a large-scale structure formation shock (Enßlin \& Brüggen 2002).

The morphology of the long linear structures that appear in frames 5 and 6 of Fig. 7 are indeed reminiscent of shock fronts. This structure would then have to be located at the periphery of the cluster. As argued in Sect. 8.3 it would probably have to be located on the near side of the cluster to prevent significant beam depolarization following the passage of the radiation through the Perseus cluster. The good alignment of the front (frame 6) and the lenticular structure (frame 7) argues that they are co-located. Do we perhaps observe the actual compression of a huge $(1 \times 0.5 \mathrm{Mpc})$ hibernating bubble by a large-scale structure formation shock?

The Perseus cluster is situated at the eastern end point of the Perseus-Pisces super cluster. The fronts and the lens are in the area where one would expect large-scale structure formation shocks from general structure formation simulations. The fact

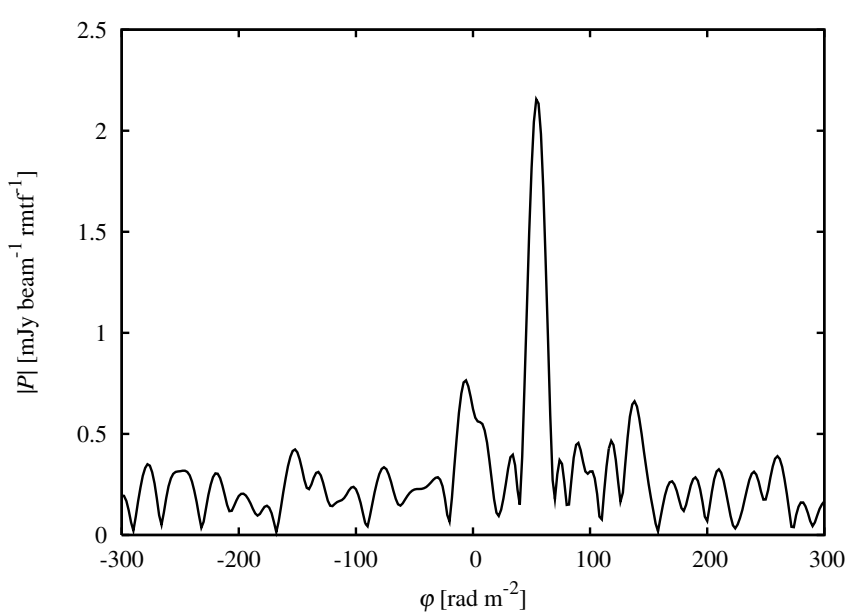

Fig. 17. Faraday depth spectrum a line-of-sight through the curved blob due north of NGC 1265.

that the front is so straight over a distance of more than $3 \mathrm{Mpc}$ is not impossible; many of the fronts in these simulations show that behaviour. Enßlin et al. (2001) report a tentative detection of a cosmological shock wave that overruns one of the radio lobes of NGC 315 . NGC 315 is positioned at the intersection of several sub filaments of the Perseus-Pisces super cluster.

The structure of the doughnut strongly resembles the structure and topology of the magnetic field in a pre-shock bubble, as simulated by Enßlin \& Brüggen (2002). Assuming the doughnut to be situated at the distance of the Perseus cluster, it measures $250 \mathrm{kpc}$ across, consistent with the hypothesis of an AGN bubble.

The blob that is visible in frame 7 of Fig. 7, north of NGC 1265, is also a good bubble candidate. It has a slightly curved shape. Its angular size is approximately $30^{\prime} \times 15^{\prime}$, which corresponds to $750 \times 375 \mathrm{kpc}$ at the distance of the cluster. It is striking that it is located just north of the steep spectrum tail of NGC 1265 and has roughly the same curvature. As can be seen in Fig. 17, its Faraday depth is approximately 50-60 $\mathrm{rad} \mathrm{m}^{-2}$. This is close to the value observed in the main part of the tail by O'Dea \& Owen (1986). Could this structure be a detached bubble associated with a previous phase of activity of NGC 1265? The projected separation of about $15^{\prime}$ translates to about $300 \mathrm{kpc}$ or 300 million years for a transverse velocity of $1000 \mathrm{~km} \mathrm{~s}^{-1}$. We have also searched for polarized emission in the steep spectrum tail of NGC 1265 (Sijbring \& de Bruyn $1998)$, but did not find any significant $\left(\gtrsim 1 \mathrm{mJy} \mathrm{beam}^{-1}\right)$ peaks.

The bar structure still further north is rather straight, but is both narrower and shorter than the two fronts on the west side of the cluster. Such structures have been discussed by Enßlin \& Brüggen (2002). With the current limited information on the detailed properties of these regions it is inappropriate to start making a detailed comparison.

\section{Remaining puzzles and future work}

Through the discovery of highly polarized, very extended and very low surface brightness emission associated with the Perseus cluster we believe we have uncovered a rich 
diagnostic tool. This tool has the potential to study the magneto-ionic medium, the relativistic plasma content, the magnetic field topology and strength and the interaction with LSS-formation related inflow into clusters of galaxies. Keshet et al. (2004) predict that cosmological shock waves should be easily detectable with the Square Kilometer Array (SKA) and the Low Frequency Array (LOFAR) at frequencies below $500 \mathrm{MHz}$. They suggest that the signal could already be marginally detectable with current instruments and they are probably correct.

In future papers, following the reduction and analysis of several recently acquired new full polarization datasets on the Perseus and other clusters, at wavelengths from $21 \mathrm{~cm}$ to $250 \mathrm{~cm}$, we will address some of these issues in considerably more detail. The detection of similar structures in other clusters would support our current hypothesis for their origin. In order to minimize complications due to high Faraday depth foreground polarization confusion, these clusters are located at high Galactic latitude. We have chosen the Coma and Abell 2256 clusters. Recently, filamentary structures have been observed in the Abell 2255 cluster (Govoni et al. 2005). The surface brightness of the structures found in Abell 2255 is about 1 to 2 orders of magnitude higher than the structures we found in or near the Perseus cluster. We expect that using RMsynthesis one could trace these structures well into the largescale filaments of the cosmic web.

On the observational side we can identify the following tasks that lie ahead of us:

- Image the corresponding total intensity and determine the spectral properties of the emission.

- Determine the polarization percentage and structure over an even wider range of frequencies. This may allow us to unravel the internal plasma density and intrinsic magnetic field structure.

- Determine where the boundaries of the interaction between cluster and large-scale structure flows in the intergalactic medium are located.

- Extend the sample of clusters

- Combine the current data with data taken at different observing frequencies.

The last point is necessary in order to derive the magnetic field direction in the plane of the sky. For this one needs the polarization angle at $\lambda=0, \chi_{0}$. Unfortunately the $1 \sigma$ error on the derived Faraday depth is too large (more than $1 \mathrm{rad} \mathrm{m}^{-2}$ ) to allow derotation to $\lambda=0$. The $3 \sigma$ error in position angle due to a $1 \sigma$ error of $1 \mathrm{rad} \mathrm{m}^{-2}$ at $\lambda_{0}^{2} \approx 0.77$ is $2.3 \mathrm{rad}$. This implies that the $99 \%$ confidence range is more than $4 / 3$ times the $180^{\circ}$ range that the position angle can assume.

On the theoretical side we are faced with the following questions:

- How can we explain the uniformity of the excess RM, in both sign and magnitude, across the face of the Perseus cluster?

- How can we distinguish between the various models for the origin of the relativistic particles at the edges of the cluster (are they from re-energized relics or are they shockaccelerated?)
- How long can AGN bubbles hibernate before they fail to be re-energized to radiate at observable radio frequencies?

- What are the observable effects if massive galaxies, or galaxy sub-groups, plough through such bubbles? The numerical simulations suggest that this would happen rather frequently.

Bubbles carry important information on the final phases of radio galaxies and the study of AGN fossils may bring us somewhat closer to an answer on the long-standing question: do radio galaxies stop being active rather suddenly or do they just fade away? The study of bubbles and relics may quantify the role played by AGN activity and its feedback on cluster evolution. Cluster evolution and radio AGN activity are clearly linked in the central parts of clusters and may provide the answer to "the cooling catastrophe" (Fabian et al. 2003; Clarke et al. 2004). Are enough bubbles blown to prevent such a catastrophe? It is believed that AGN activity is recurrent (Schoenmakers et al. 2000) but it is at this moment unclear how many bubbles exist in a cluster, nor do we know how long they can hibernate. Is the large-scale polarized structure that arches around the steep spectrum tail of NGC 1265 indeed the remains of an earlier phase of activity?

\section{Conclusions and summary}

This paper has described low frequency observations of the Perseus cluster obtained with the WSRT. The total intensity data agree with earlier observations and do not present new insights into the cluster emission. However, using a novel method of wide band polarimetric imaging, called RM-synthesis, we have been able to extract information on extremely faint polarized signals buried deep in the noise of individual narrow band frequency images.

Polarized emission has been detected in the Perseus cluster over a wide range of Faraday depths from about 0 to $90 \mathrm{rad} \mathrm{m}^{-2}$. Low $\phi$ emission $\left(\phi<15 \mathrm{rad} \mathrm{m}^{-2}\right)$ is attributed to the local Galactic foreground. Emission at values of $\phi>$ $30 \mathrm{rad} \mathrm{m}^{-2}$ on the other hand, shows organized structures on scales up to a degree and displays rapidly fluctuating polarization angles on scales of the order of $1^{\prime}-2^{\prime}$. A Galactic foreground interpretation for the high $\phi$ emission appears extremely implausible. The diffuse structures have a clear excess Faraday depth of about $+40 \mathrm{rad} \mathrm{m}^{-2}$ relative to a dozen distant radio galaxies surrounding the Perseus cluster and are probably located at the near side periphery of the cluster.

Most of the polarized emission, which is located at distances of $0.5-1.5$ from the cluster centre, appears 1-2 orders of magnitude too bright to be explainable as Thomson scattered emission of the central radio source off the thermal electrons in the cluster (Syunyaev 1982). However, this remains a viable explanation for previously detected highly polarized $21 \mathrm{~cm}$ emission from the inner $10^{\prime}-20^{\prime}$ as well as part of the $81-95 \mathrm{~cm}$ emission observed in the region surrounding NGC 1275. We believe that the bulk of the emission associated with the Perseus cluster is related to buoyant bubbles of relativistic plasma, probably relics from still active or now dormant AGN within the cluster. A lenticular shaped structure, 
referred to as the lens, and measuring $0.5-1 \mathrm{Mpc}$ is strikingly similar to the structures predicted by Enßlin \& Gopal-Krishna (2001) and may be in the process of being re-energized. A bright, about $0.4 \mathrm{Mpc}$ sized, structure located to the north of the steep spectrum tail of NGC 1265 may be evidence of an even earlier phase of activity from this famous head-tail radio galaxy (Sijbring \& de Bruyn 1998).

We have not yet detected the total intensity corresponding to any of these polarized structures. This may be due to a combination of dynamic range in Stokes $I$ and lack of short spacing sensitivity. However, there is no doubt that the structures are very highly polarized $(>20 \%)$, as is common for many relic sources in clusters.

At the western edge of the cluster we have detected linear structures of several Mpc length that may be related to shocks caused by infall of gas into the Perseus cluster along the Perseus-Pisces filamentary structure of the cosmic web (Enßlin et al. 1998; Burns 1998).

The ability of low-frequency RM-synthesis to image very extended polarized structures, at intensity levels far below the total intensity image which may be limited by confusion or dynamic range problems, makes it a very powerful technique. Regions of low surface brightness and low magnetoionic plasma density are predicted to occur at the periphery of clusters and extending into the inter cluster medium and the interface with regions shocked in the process of large-scale structure formation. The radio spectra associated with such emission are often (predicted to be) steep requiring observations at low frequencies. The Low Frequency Array (LOFAR, see http://www.lofar.org) is the ideal instrument to embark on such studies. It combines excellent surface brightness sensitivity and a wide frequency range with the capability to perform high angular resolution studies in cases where beam depolarization is important.

Acknowledgements. The Westerbork Synthesis Radio Telescope is operated by ASTRON (Netherlands Foundation for Research in Astronomy) with support from the Netherlands Foundation for Scientific Research (NWO). The Wisconsin H-Alpha Mapper is funded by the National Science Foundation.

\section{References}

Bower, G. C., Backer, D. C., Zhao, J., Goss, M., \& Falcke, H. 1999, ApJ, 521, 582

Brüggen, M. 2003, ApJ, 592, 839

Brentjens, M. A., \& de Bruyn, A. G. 2005, A\&A, 441, 1217

Burn, B. J. 1966, MNRAS, 133, 67

Burns, J. O. 1998, Science, 280, 400

Carr, J. C., Beatson, R. K., Cherrie, J. B., et al. 2001, in SIGGRAPH 2001, Computer Graphics Proceedings, ed. E. Fiume (ACM Press / ACM SIGGRAPH), 67-76

Cen, R., \& Ostriker, J. P. 1999, ApJ, 514, 1

Churazov, E., Forman, W., Jones, C., \& Böhringer, H. 2003, ApJ, 590, 225

Clarke, T. E., Blanton, E. L., \& Sarazin, C. L. 2004, ApJ, 616, 178

Clarke, T. E., Kronberg, P. P., \& Böhringer, H. 2001, ApJ, 547, L111

Condon, J. J., Cotton, W. D., Greisen, E. W., et al. 1998, AJ, 115, 1693

Conway, R. G., \& Strom, R. G. 1985, A\&A, 146, 392

de Bruyn, A. G. 1995, Unpublished de Bruyn, A. G. 1996, RM-synthesis via wide-band low-frequency polarimetry, Tech. Rep. NFRA Note 655, ASTRON, Dwingeloo

Enßlin, T. A., \& Brüggen, M. 2002, MNRAS, 331, 1011

Enßlin, T. A., \& Gopal-Krishna. 2001, A\&A, 366, 26

Enßlin, T. A., Biermann, P. L., Klein, U., \& Kohle, S. 1998, A\&A, 332, 395

Enßlin, T. A., Simon, P., Biermann, P. L., et al. 2001, ApJ, 549, L39

Fabian, A. C., Sanders, J. S., Allen, S. W., et al. 2003, MNRAS, 344, L43

Gaensler, B. M., Dickey, J. M., McClure-Griffiths, N. M., et al. 2001, ApJ, 549, 959

Govoni, F., Feretti, L., Giovannini, G., et al. 2001, A\&A, 376, 803

Govoni, F., Murgia, M., Feretti, L., et al. 2005, A\&A, 430, L5

Haffner, L. M., Reynolds, R. J., Tufte, S. L., et al. 2003, ApJS, 149, 405

Hamaker, J. P., Bregman, J. D., \& Sault, R. J. 1996, A\&AS, 117, 137

Harris, F. J. 1978, in Proc. IEEE, Volume 66, p. 51-83, 51-83

Haverkorn, M. 2002, Ph.D. Thesis, Leiden University

Haverkorn, M., Katgert, P., \& de Bruyn, A. G. 2003a, A\&A, 403, 1045

Haverkorn, M., Katgert, P., \& de Bruyn, A. G. 2003b, A\&A, 403, 1031

Johnston-Hollitt, M. 2004, in The Riddle of Cooling Flows in Galaxies and Clusters of galaxies

Johnston-Hollitt, M., Hollitt, C. P., \& Ekers, R. D. 2004, in The Magnetized Interstellar Medium, 13

Kaastra, J. S., Lieu, R., Tamura, T., Paerels, F. B. S., \& den Herder, J. W. 2003, A\&A, 397, 445

Keshet, U., Waxman, E., \& Loeb, A. 2004, ApJ, 617, 281

Killeen, N. E. B. 1998, Unpublished

Leahy, J. P. 1987, MNRAS, 226, 433

Miley, G. K., Perola, G. C., van der Kruit, P. C., \& van der Laan, H. 1972, Nature, 237, 269

Miniati, F., Ryu, D., Kang, H., et al. 2000, ApJ, 542, 608

Mitra, D., Wielebinski, R., Kramer, M., \& Jessner, A. 2003, A\&A, 398, 993

Napier, P. J. 1999, in Synthesis Imaging in Radio Astronomy, ASP Conf. Ser., 180, 37

Navarro, J., de Bruyn, A. G., Frail, D. A., Kulkarni, S. R., \& Lyne, A. G. 1995, ApJ, 455, L55

O’Dea, C. P., Dent, W. A., \& Balonek, T. J. 1984, ApJ, 278, 89

O'Dea, C. P., \& Owen, F. N. 1986, ApJ, 301, 841

O’Dea, C. P., \& Owen, F. N. 1987, ApJ, 316, 95

Quilis, V., Ibanez, J. M. A., \& Saez, D. 1998, ApJ, 502, 518

Rottgering, H. J. A., Wieringa, M. H., Hunstead, R. W., \& Ekers, R. D. 1997, MNRAS, 290, 577

Rybicki, G. B., \& Lightman, A. P. 1979, Radiative Processes in Astrophysics (John Wiley \& Sons)

Sault, R. J., Hamaker, J. P., \& Bregman, J. D. 1996, A\&AS, 117, 149

Schoenmakers, A. P., de Bruyn, A. G., Röttgering, H. J. A., van der Laan, H., \& Kaiser, C. R. 2000, MNRAS, 315, 371

Sijbring, D., \& de Bruyn, A. G. 1998, A\&A, 331, 901

Sijbring, L. G. 1993, Ph.D. Thesis, Groningen University

Simonetti, J. H., \& Cordes, J. M. 1986, ApJ, 310, 160

Simonetti, J. H., Cordes, J. M., \& Spangler, S. R. 1984, ApJ, 284, 126

Spergel, D. N., Verde, L., Peiris, H. V., et al. 2003, ApJS, 148, 175

Strom, R. G., \& Conway, R. G. 1985, A\&AS, 61, 547

Struble, M. F., \& Rood, H. J. 1999, ApJS, 125, 35

Syunyaev, R. A. 1982, Pis ma Astronomicheskii Zhurnal, 8, 323

Weisberg, J. M., Cordes, J. M., Kuan, B., et al. 2004, ApJS, 150, 317

Wieringa, M. H. 1992, Experimental Astronomy, 2, 203

Wieringa, M. H., de Bruyn, A. G., Jansen, D., Brouw, W. N., \& Katgert, P. 1993, A\&A, 268, 215

Wise, M. W., \& Sarazin, C. L. 1990, ApJ, 363, 344

Wise, M. W., \& Sarazin, C. L. 1992, ApJ, 395, 387 